\title{
NATUURKUNDIGE WOORDENLIJST.
}

$f$ beteekent fructus, vrucht.

a 》 arbor, de boom of plant.

Zie verder de noten, achter deze woordenlijst.

Achter de wetenschappelijke namen ontbreken die der ontdekkers of naamgevers. In de hulpmiddelen, in Indie ter mijne beschikking, vond ik ze niet. 


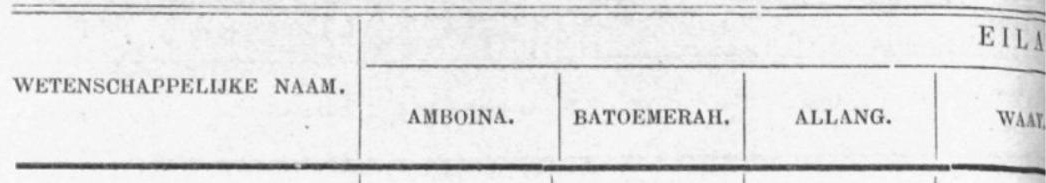

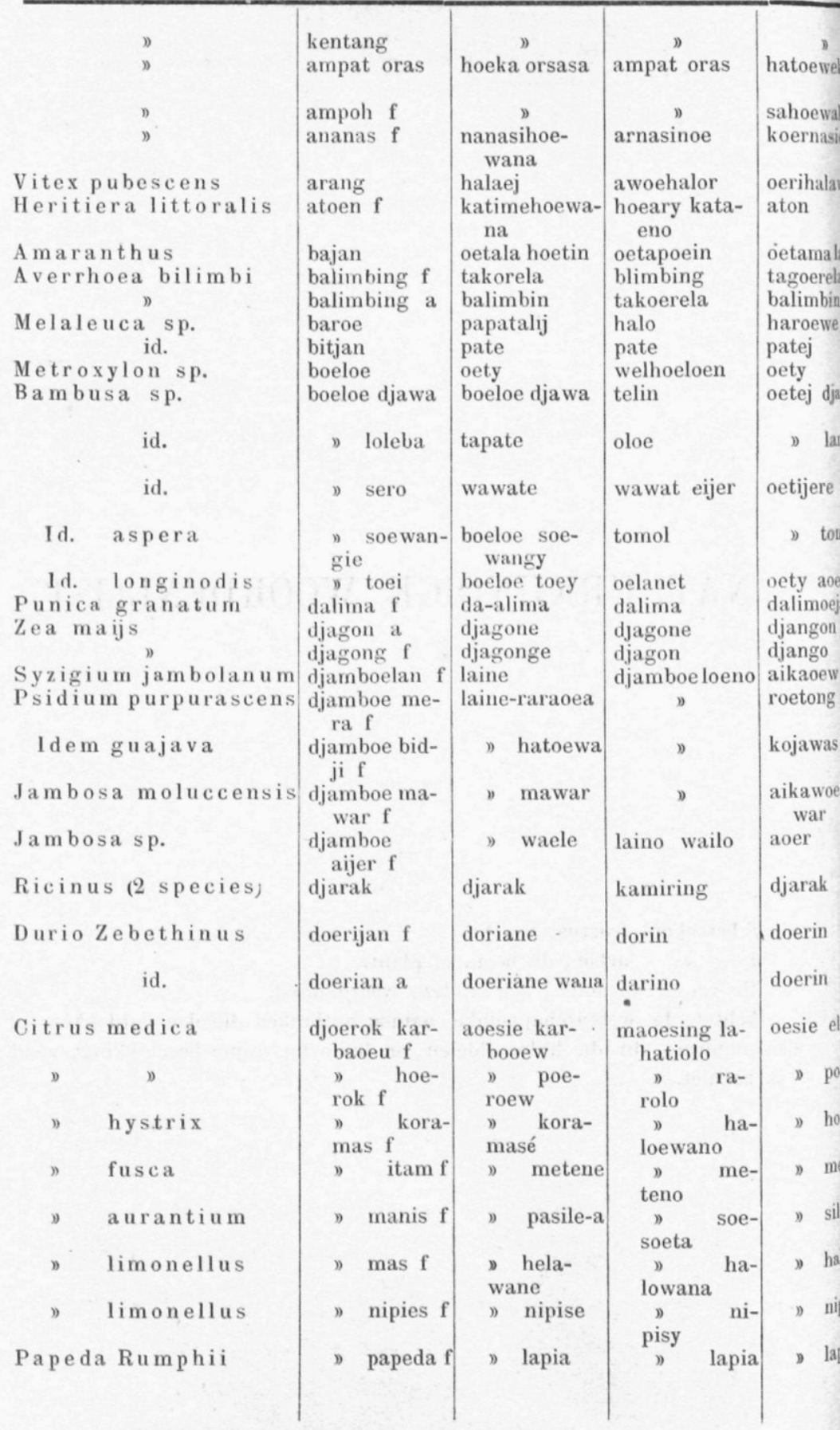




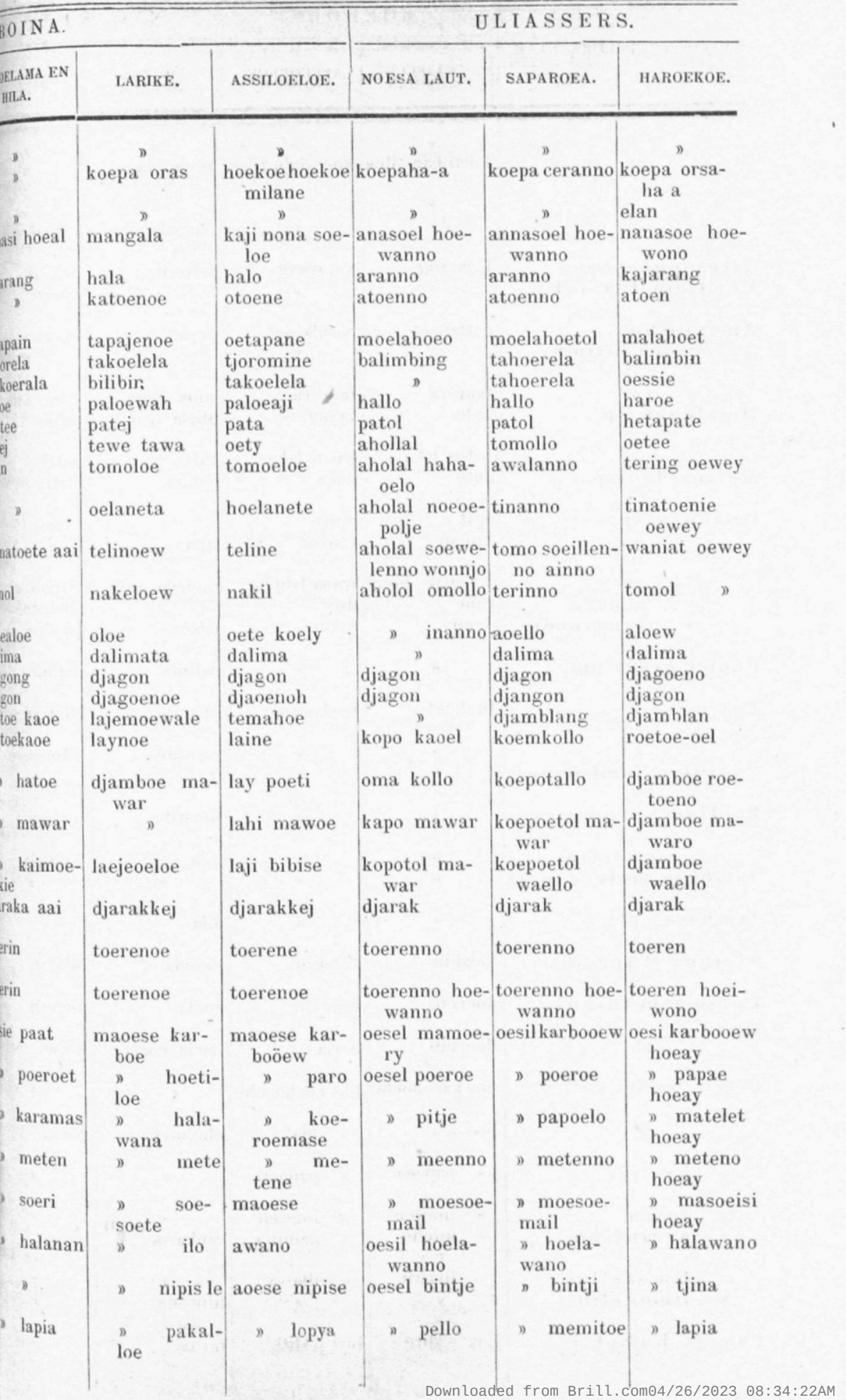




\begin{tabular}{|c|c|c|c|c|}
\hline \multirow{2}{*}{ WETENSCHAPPELIJKE NAAM. } & \multicolumn{2}{|c|}{ BOEROE } & \multicolumn{2}{|c|}{ N. 0. K UST V } \\
\hline & KAJELI. & ALEFOEROE. & НОТТI. & HATOEV \\
\hline $\begin{array}{l}n \\
n\end{array}$ & $\stackrel{n}{n}$ koemban tjlna & koemban tjina & aioemina & ) \\
\hline ) & 》 & 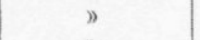 & ) & $y$ \\
\hline $\begin{array}{l}\text { Vitex pubescens } \\
\text { Heritiera littoralis }\end{array}$ & kajarang & $\underset{n}{\text { kararang }}$ & $\begin{array}{l}\text { phoeala aina- } \\
\text { sia } \\
\text { ariaran } \\
\end{array}$ & $\begin{array}{l}\text { hoean sisi } \\
\text { ariaran } \\
\text { hoean }\end{array}$ \\
\hline $\begin{array}{l}\text { Amaranthus } \\
\text { Averrhoeabilimbi }\end{array}$ & $\underset{\text { oetlaboet }}{\text {. }}$ & oetalasoet & pepa & malanoe \\
\hline Melaleuca sp. & $\begin{array}{l}\text { taprera } \\
\text { balo }\end{array}$ & $\begin{array}{l}\text { taprera } \\
\text { tagoe }\end{array}$ & $\begin{array}{l}\text { weta-weta } \\
\text { phala (pari) }\end{array}$ & $\begin{array}{l}\text { wita wita } \\
\text { paloe }\end{array}$ \\
\hline Metroxylon sp. & $\begin{array}{l}\text { batin laka } \\
\text { oete }\end{array}$ & $\begin{array}{l}\text { batin laka } \\
\text { oeka }\end{array}$ & $\begin{array}{l}\text { batta } \\
\text { ephita }\end{array}$ & $\begin{array}{l}\text { patti } \\
\text { alati }\end{array}$ \\
\hline Bambusa sp. & $\begin{array}{l}\text { epit } \\
\text { teman }\end{array}$ & $\begin{array}{l}\text { epite } \\
\text { teman }\end{array}$ & tiha & $\begin{array}{l}\text { kapale } \\
\text { tipo }\end{array}$ \\
\hline $\begin{array}{ll} & \text { aspera } \\
\text { " longinodis }\end{array}$ & $\begin{array}{l}\text { wanatte hony } \\
\text { rene } \\
\text { aoen }\end{array}$ & $\begin{array}{l}\text { wana lahene } \\
\text { diry } \\
\text { netat }\end{array}$ & $\begin{array}{l}\text { soehala wala } \\
\text { tibone }\end{array}$ & $\begin{array}{l}\text { alatian ait } \\
\text { allahatoe } \\
\text { tipoloe }\end{array}$ \\
\hline Punica granatum & $n$ & 》 & dalima & dalima \\
\hline Zea maijs & beskoetoe & beskoetoe & phasanina & allomaoe \\
\hline Cancos & $"$ & ) & pasanina & allamahoe \\
\hline Syzigium jambolanum & 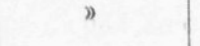 & 3 & n & ) \\
\hline Psidium purpurascens & $n$ & $n$ & moetiha & makoei \\
\hline g guajava & 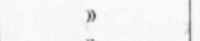 & ) & aila & poppoti \\
\hline Jambosa moluccensis & 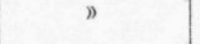 & ) & $n$ & (1) \\
\hline Jambosa sp. & ) & 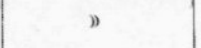 & aila & ) \\
\hline Ricinus (2 species) & kalakin & kalakin & eimalate & eilatoe \\
\hline Durio zebethinus & doerian & doerian & roela & tooean \\
\hline id. & doerian & oeria & oeria roela & hoean tor \\
\hline Citrus medica & hos karbooehe & hos karbooche & 》 & \\
\hline ) & hos lea & »lea & ehehoetina & poein \\
\hline bystrix & D papoea & ) papoea & ) & 》 \\
\hline ) fusca & ) imeten & " imeten & ”. & \\
\hline ) aurantium & ) minae & D damina & sankaria & pipise \\
\hline " limonellus & " illawa & n illawa & 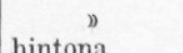 & y \\
\hline Papeda Rumphii & hus walite & hos walite & mitoa & , \\
\hline
\end{tabular}




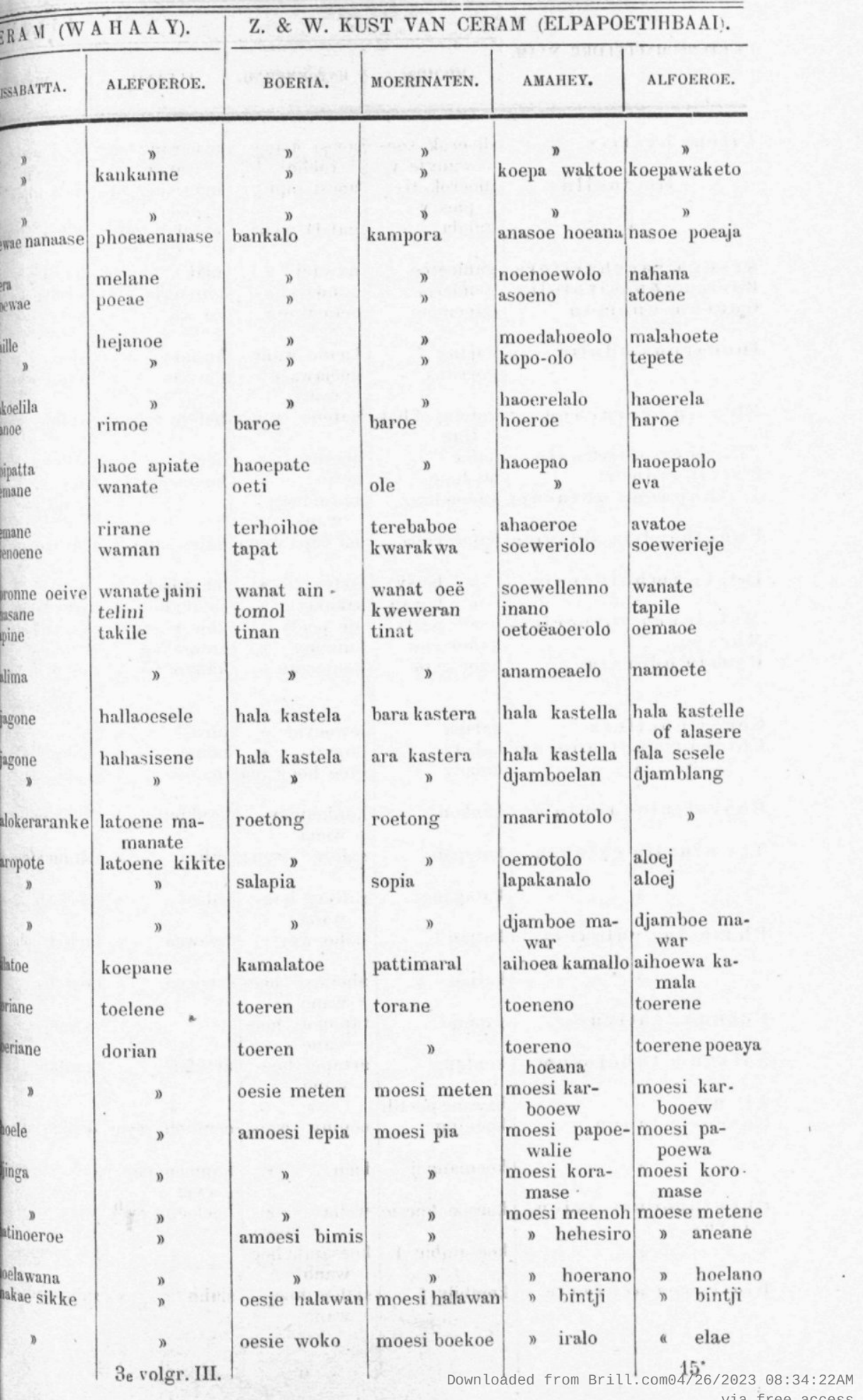




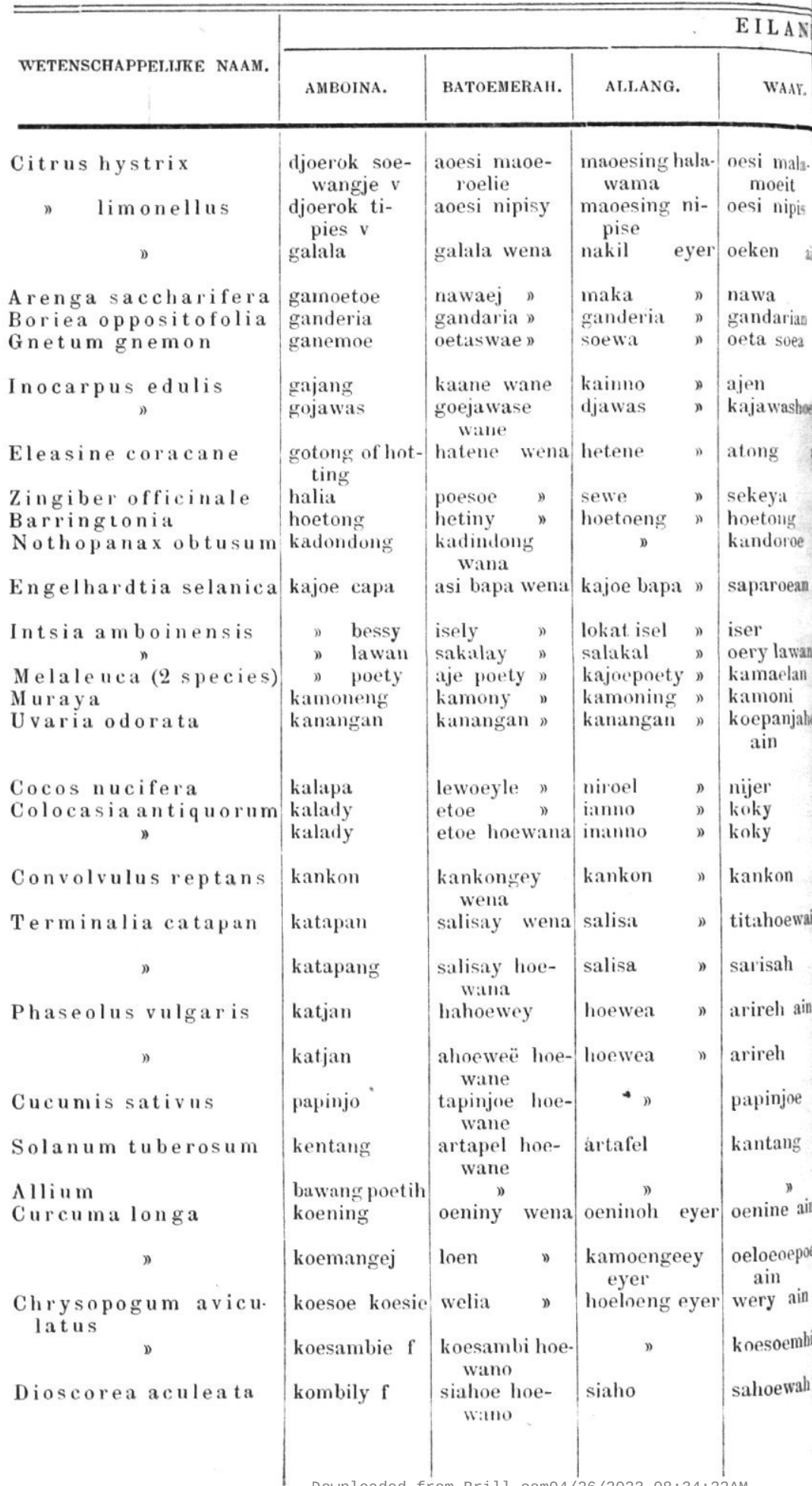




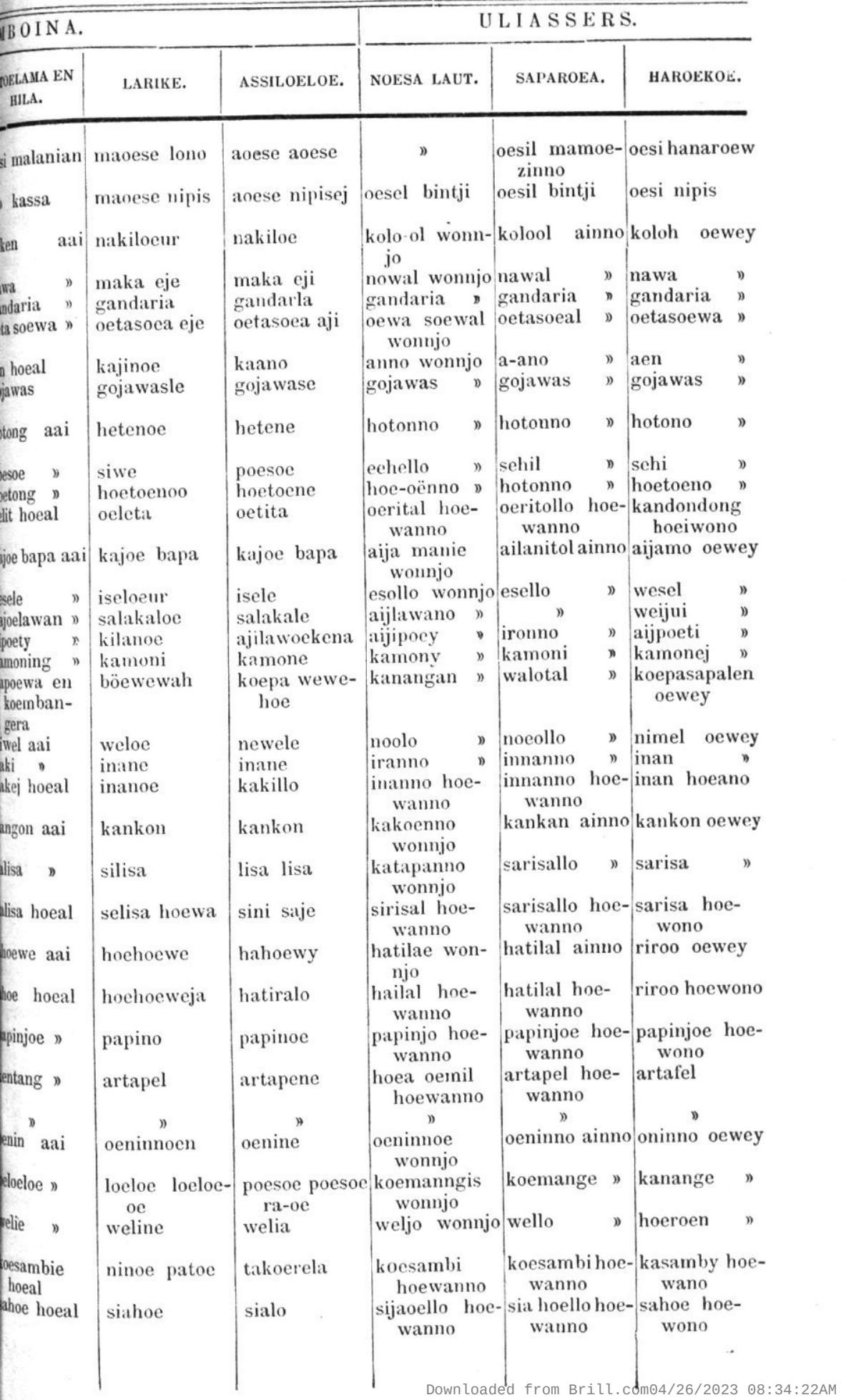




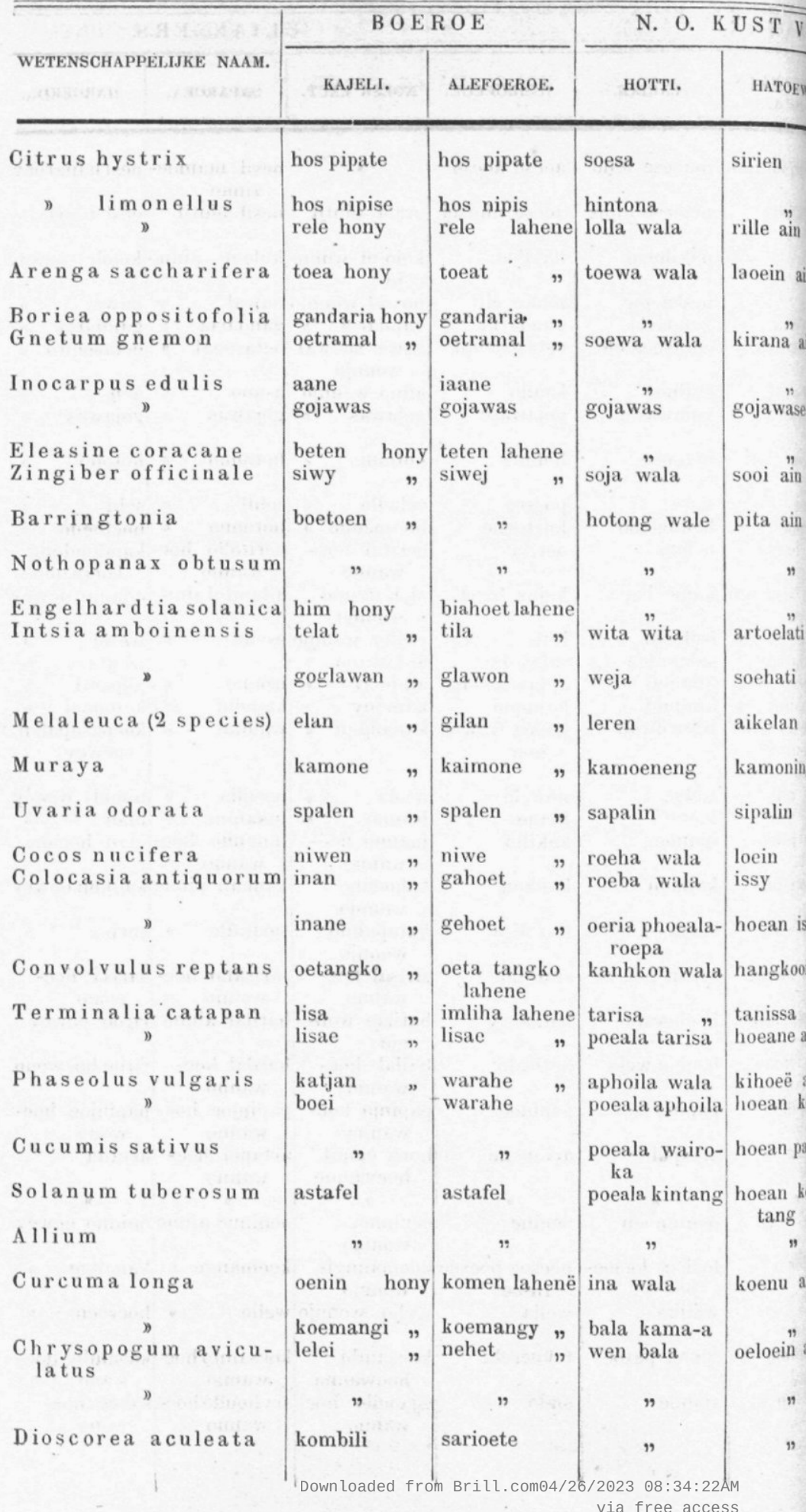




\section{ERA I (W A H A A Y).}

ALEFOEROE.

ALEFOEROE. BOERIA.

-

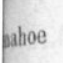

skas sikke

gniene oewe

awa

aini

awa jaini

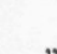

wassa" jain

" somone

ewe

etele oewe

$n$

11

oewe

epaoeti jaine

sepi jaine

loehoete jaine

$\eta$

oerit

Jakane

elan

amoniene

ipalwene ,

iwene

nane

towae inane

anko oewe

anissa oewe

vewae aanisa

thoeë oewe

wewae ka-

hoeë

bewae bi-

lonka

"

enir

"

narin

Z. \& W. KUST VAN CERAM (ELPAPOETIHBAAI) 


\begin{tabular}{|c|c|c|c|c|}
\hline \multirow{2}{*}{ WETENSCHAPPELIJKE NAAM } & \multicolumn{4}{|r|}{ EILAI } \\
\hline & AMBOINA. & BATOEMERAH. & ALLANG. & WAAY, \\
\hline Dioscorea aculeata & konto konto & $\begin{array}{l}\text { walehaoewe- } \\
\text { toely wena }\end{array}$ & $\begin{array}{l}\text { walihoetoeata } \\
\text { eyer }\end{array}$ & $\begin{array}{l}\text { haoewetoe } \\
\text { ain }\end{array}$ \\
\hline Cucurbita melopepo & laboe & laboene wena & laboe eyer & raboewe: \\
\hline Lansium domesticum & $\begin{array}{l}\text { lansa } \\
\text { lansa }\end{array}$ & $\begin{array}{l}\text { lasata } \\
\text { lasate hoe- } \\
\text { wano }\end{array}$ & $\begin{array}{ll}\text { poewan } & , \\
\text { pocano } & "\end{array}$ & $\begin{array}{l}\text { aypoewa } \\
\text { aipoewan }\end{array}$ \\
\hline Myristica iners & laka & laka wena & ala ala $"$, & alahalan a \\
\hline Alpinia galanga & langkoewas & lakoewase , & lakoewase , & lawassy \\
\hline Pterocarpus indicus & lengoewa & lala, & lala ", & nala \\
\hline Citrus & $\begin{array}{l}\text { lemon } \\
\text { limon } f\end{array}$ & $\begin{array}{l}\text { aoese } \\
\text { aoesi hoewano }\end{array}$ & $\begin{array}{l}\operatorname{masa} \\
\text { maoese }\end{array}$ & $\begin{array}{l}\text { oesy } \\
\text { oesy }\end{array}$ \\
\hline $\begin{array}{l}\text { Borassus flabellifor- } \\
\text { mis }\end{array}$ & lontar & lontar wena & lontar ayer & saliboe ai \\
\hline Mangifera & manga & ambalamoe & manga $\quad$, & ambalan , \\
\hline 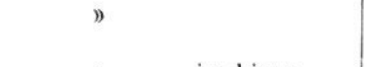 & manga f & $\begin{array}{c}\text { ambalamoe } \\
\text { hoewane }\end{array}$ & manga & ambalan \\
\hline indica & $"$ dolol f & $\begin{array}{l}\text { ambalamoe } \\
\text { dodole }\end{array}$ & $"$ koeha & manga do \\
\hline foetida & $" \quad$ koiry $\mathrm{f}$ & wine koe- & $"$ koinnie & nie ko \\
\hline$n$ & $" \quad$ paoe f & $"$ paoe & " paoe & $" \quad p$ \\
\hline Bruguiera Rumphii & mange mange & wate wena & $\begin{array}{l}\text { mange mange } \\
\text { eyer }\end{array}$ & watah aif \\
\hline Carcinia mangoestana & mangoestan $\mathrm{f}$ & $\begin{array}{l}\text { mangoestan } \\
\text { hoeana }\end{array}$ & mangoestan & mangoesta \\
\hline Jasminum sambac & manor & $\begin{array}{l}\text { baugoeloe } \\
\text { wena }\end{array}$ & $\begin{array}{l}\text { manoeloe } \\
\text { eyer }\end{array}$ & koepapoety \\
\hline Cynometra & natm natm f & $\begin{array}{c}\text { namoe namoe } \\
\text { hoewanoe }\end{array}$ & lamoeta & lamoet \\
\hline ) & namoe namoe & $\begin{array}{l}\text { lamoe lamoe } \\
\text { wena }\end{array}$ & lamoeta eyer & lamoet ai \\
\hline $\begin{array}{l}\text { Artocarpus integri- } \\
\text { folia }\end{array}$ & nangka & ana-a wena & nanka $\quad$, & anaan \\
\hline 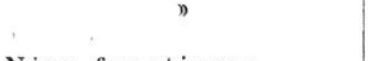 & rangka f & $\begin{array}{l}\text { nanka hoe- } \\
\text { wana }\end{array}$ & nanka & ana-an \\
\hline Nipa fructicosa & nipa & paleney wena & palean eyer & parinan a \\
\hline Areca nibung & njibon & njibon & njibon & paroe \\
\hline$\eta$ & nona & $"$ & $"$ & maboei \\
\hline Dioscorea & $\begin{array}{l}\text { oeby } \\
\text { oebie }\end{array}$ & $\begin{array}{l}\text { oehey wena } \\
\text { oehi hoewana }\end{array}$ & $\begin{array}{l}\text { oehej eeyer } \\
\text { oehoe }\end{array}$ & $\begin{array}{l}\text { hery ain } \\
\text { herie }\end{array}$ \\
\hline Oryza sativa & pady & alotoeny wena & $\begin{array}{l}\text { alaetoewa } \\
\text { eyer }\end{array}$ & alaoetoet \\
\hline Momordica & papari & papiriany " & papare eyer & paparyan \\
\hline$n$ & papary f & papiliano & papary & paparyan \\
\hline 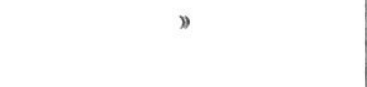 & parapat & parapat wena & parapit eyer & $\begin{array}{l}\text { wata koep } \\
\text { ain }\end{array}$ \\
\hline & Down loaded & om Brill.come4 & $26 / 2023 \quad 08: 34$ & \\
\hline
\end{tabular}




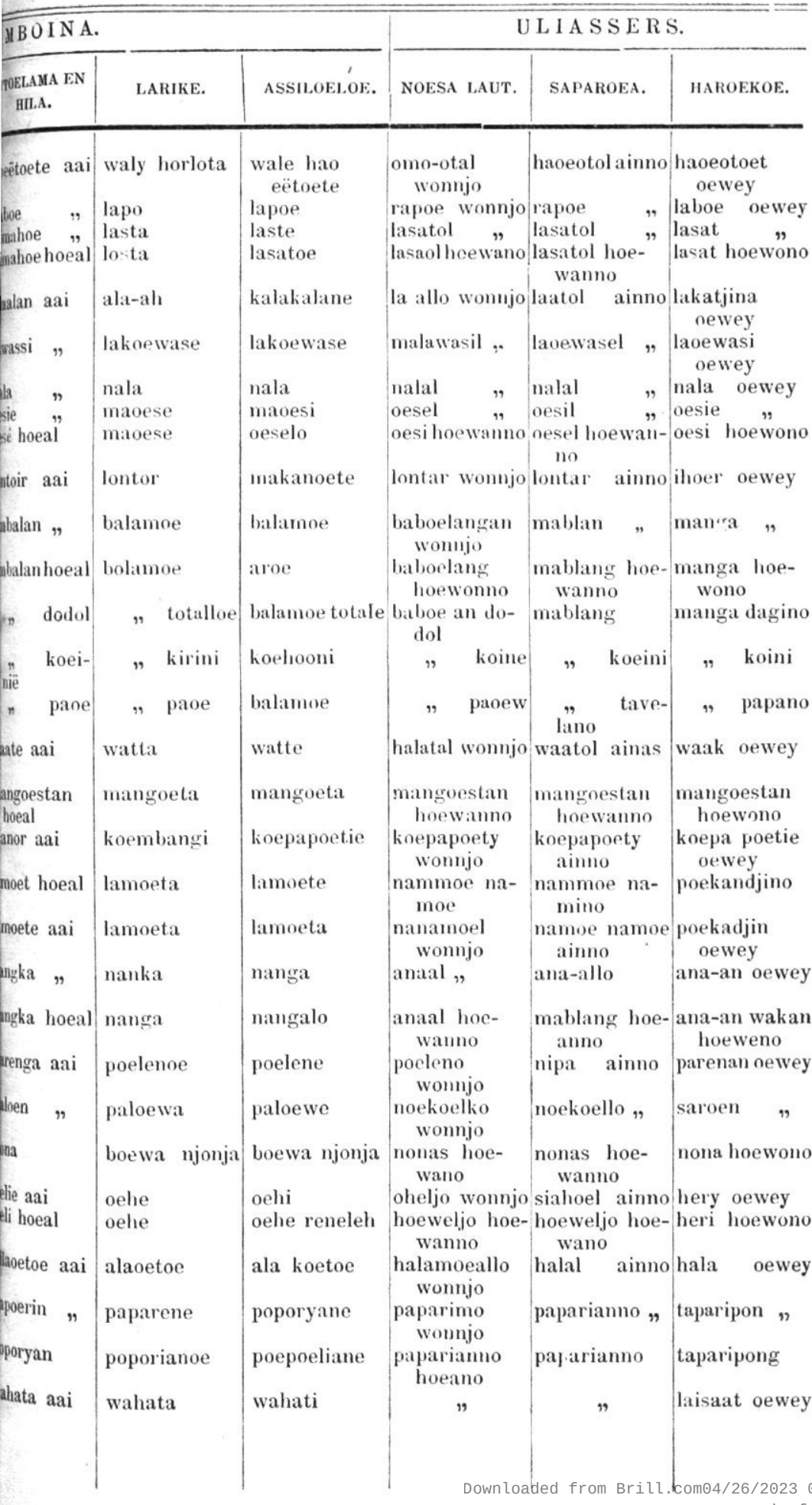




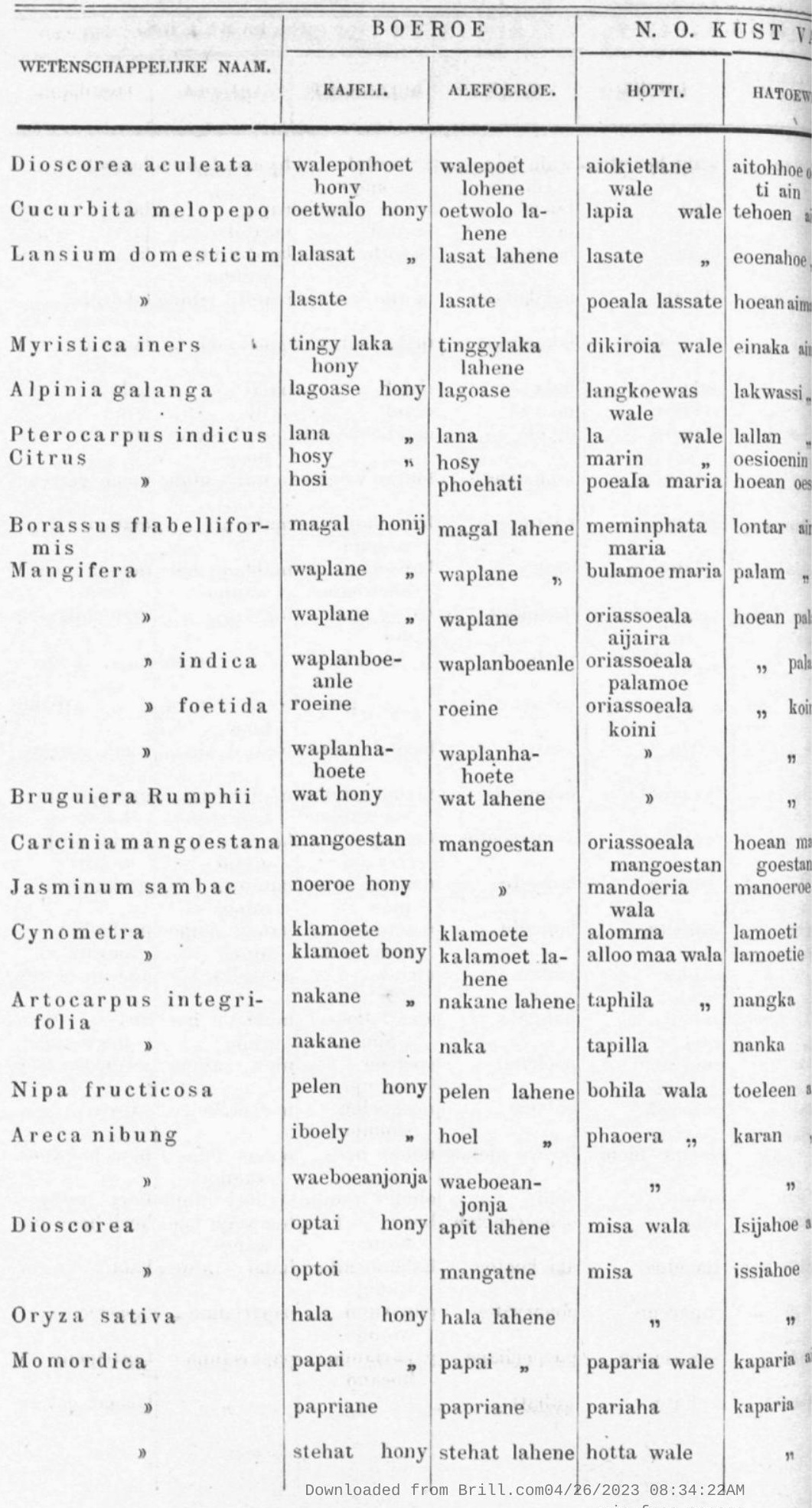




\section{RA M (WA HA A Y).}

\begin{tabular}{l|l}
\hline uSSABATTA. & ALFOEROE.
\end{tabular}

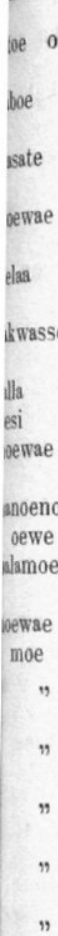

ve oewe

boe $"$

isate $"$

pewae nacate

las oewe

kwasse "

illa

esi " "

Dewae oesi

anoenoete

oewe

alamoe oewe

wae bala-

s

n

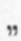

$n$

"

nanoeroe

oewe

amoete

moete oewe

anga

beneene

oewe

koene oewe

n

che oewe

siahoe

ท

repare oewe hahanoe jaine pipar

laboe
nasate
hoewoe nasate
loeëpepi jaine
nala la
oesnoesi
poeae moessi

laboe
noewate nasate
loeëpepi jaine
nala
oesnoesi "
poeae moessi

laboe
noewate nasate
loeëpepi jaine
nala
oesnoesi "
poeae moessi

kapi jaine

haoe $"$

lan

lamoete

koelene jaine

janitte jaine

janite
Z. \& W. KUST VAN CERAM (ELPAPOETIHBAAI).

\begin{tabular}{l|l|l|l}
\hline BOERIA. & MOERINATEN. & AMAHEY. & ALFOEROE.
\end{tabular}

poewae papa- haoen

\section{nala "}

oesi amoesi

\section{AHEY.}

ALFOEROE.

\begin{tabular}{c|c} 
haoestolo & taoeneetoete \\
hoero &
\end{tabular}

\section{$\operatorname{ain}$}

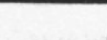

19

n

lapoero oewo- lapoe

no

lakaolo , nasate

lakaoh hoeana nasate poeaja

laset

alaat ain lakat oeẽ

lalalaano

oewono

kooerola lawasie

oewono

nara " nala oewono nala

\begin{tabular}{l|l} 
moesi \\
moesi
\end{tabular}

moesiro hoe-
ana

hoerojo ifoele

mabalang oe- mapoelang

wono

mablang hoe- wapoelan

ana

mablang teto-mablang to-

ne hoeana tore

.. koeini " koeini

hoeana

" baolo " paolo

hoeana

" lahata- lahato

olo hoeana

mangastan mangoestan

hoeana

koepa poeiro koepapoety oewono

anamoeaelo namoete

koepoety

lamoet ain lamoet oeë

oemolo oewo-namoete

amanaalo

tafela

amanaaloh tapela hoea-

hoeana kano

anaan

noeka

aie ama oewo

aia amaoe

no

noekor ain moetjoel oeë

noesoerolo

baloeë

$"$

"

nona hoeana nona

ahoehoe elang oehi

ain

ahoehoe

awoewoe

hala

"

oehero oeherie

oehero hoeana werah

hala oewono hala

paparijanno paparyana

papariane ain paparian oeë

papariane paparian paparijano

paparyane

parapat

parapat 


\begin{tabular}{|c|c|c|c|c|}
\hline \multirow[b]{2}{*}{ WETENSCHAPPELIJKE NAAM. } & \multicolumn{2}{|c|}{ 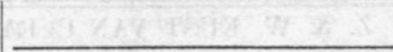 } & \multicolumn{2}{|c|}{ EILA) } \\
\hline & AMBOINA. & BATOEMERAH. & ALLANG. & WAA \\
\hline Convolvulus batatas & patatas & patatase & kastela eijer & bartoel \\
\hline I de m & patatas $\mathbf{f}$ & $\begin{array}{l}\text { patatase hoe- } \\
\text { wana }\end{array}$ & kastela & bartoekal \\
\hline Parkia & pateh & $\begin{array}{l}\text { djamaka (pe- } \\
\text { teka) hoe- } \\
\text { wana }\end{array}$ & pateka & petih \\
\hline D & . & 17 & , & $y$ \\
\hline ) & pinang & hoewa wena & hoewa eijer & hoewa : \\
\hline A reca & pinang $f$ & hoea hoewana & hoea & hoewa \\
\hline I de m (meerdere species) & pinang $\mathbf{f}$ & pitja pirin & pitja pirin & x \\
\hline Musa paradisica & pisang & temaej wena & oere eijer & hoela ain \\
\hline Nephelium lappaceum & $\underset{n}{\text { ramboetang } \mathrm{f}}$ & ramboetange & ) & $\underset{n}{\text { ramboetan }}$ \\
\hline Sapindus rarak & saboen $f$ & $\begin{array}{l}\text { sabong hoe- } \\
\text { wana }\end{array}$ & boea sabon & sambon \\
\hline Metroxylon & sagoe & lapia wena & lapial eijer & lapia \\
\hline Abrus praecatorius & saga & sagau & saga & ailaroe \\
\hline Salacca edulis & salak & salak " & salak & salak \\
\hline $\begin{array}{l}\text { Andropogon schoe- } \\
\text { nanthus }\end{array}$ & $\begin{array}{l}\text { samama } \\
\text { saré }\end{array}$ & $\begin{array}{l}\text { sasamaey } \\
\text { hisa hisa }\end{array}$ & $\begin{array}{l}\text { samama } \\
\text { hisa hisa }\end{array}$ & $\begin{array}{l}\text { samama } \\
\text { hisajeh }\end{array}$ \\
\hline Gynandropsis & sasawy & sasaweey ” & sasawej 》 & sasawy, \\
\hline Chavica betle & $\operatorname{sir}$ & kamoe $\quad$, & kanloewn v & amoew \\
\hline Calophylum sulatri & soelatie & hataoely wasy & halaoetyr & $\begin{array}{l}\text { hatoewao } \\
\text { ain }\end{array}$ \\
\hline Artocarpus incisa & soekoen f & $\begin{array}{l}\text { soene hoe- } \\
\text { wana }\end{array}$ & soe--oeno & soeoen \\
\hline Anona muricata & soerzak f & n & D & $\begin{array}{l}\text { ana-an wa } \\
\text { landa }\end{array}$ \\
\hline Tamarindus indica & tamarhinde & $\begin{array}{l}\text { makoeninoe } \\
\text { djawa }\end{array}$ & 》 & an sam \\
\hline Bambusa Teba & teboe teboe & $\begin{array}{l}\text { teboe teboeey } \\
\text { wena }\end{array}$ & toetohoel eijer & tatipan \\
\hline Artocarpus polyphema & $\begin{array}{l}\text { tjandana } \\
\text { tjampada-a }\end{array}$ & $\begin{array}{l}\text { tjandana wena } \\
\text { na-a na-a hoe } \\
\text { wana }\end{array}$ & $\begin{array}{l}\text { tjandana } \\
\text { anaa }\end{array}$ & $\begin{array}{l}\text { aisaroen } \\
\text { wassie }\end{array}$ \\
\hline Talauma Rumphii & tjampaka & $\begin{array}{l}\text { tjampakaey } \\
\text { wena }\end{array}$ & tjampaka eijer & anaan \\
\hline Kaempferia galanga & tjankor & asaoely wena & sooel & oempa \\
\hline $\begin{array}{l}\text { Caryophyllum gallanga } \\
\text { id. aromaticum }\end{array}$ & tjenkey & $\begin{array}{l}\text { oekalawanej } \\
\text { wena }\end{array}$ & pakalawan & poekalan \\
\hline Capsicum annum & tjily & maalise wena & tjily & tjily \\
\hline Kaempferia pandurata & $\begin{array}{r}\text { toemboe } \\
\text { kontji }\end{array}$ & $\begin{array}{l}\text { toemboe } \\
\text { kontji wena }\end{array}$ & $\begin{array}{l}\text { toemboe } \\
\text { kontji eijer }\end{array}$ & s \\
\hline $\begin{array}{l}\text { Saccharum officina- } \\
\text { rum }\end{array}$ & toboe & tehoena & oeteho & tehoe \\
\hline Bruguiera Rumphii & tonky & kakily & poeloeen & tonge \\
\hline Ficus benjamina & waringin & noemeoew 》 & noenoeawa & noenoew \\
\hline
\end{tabular}




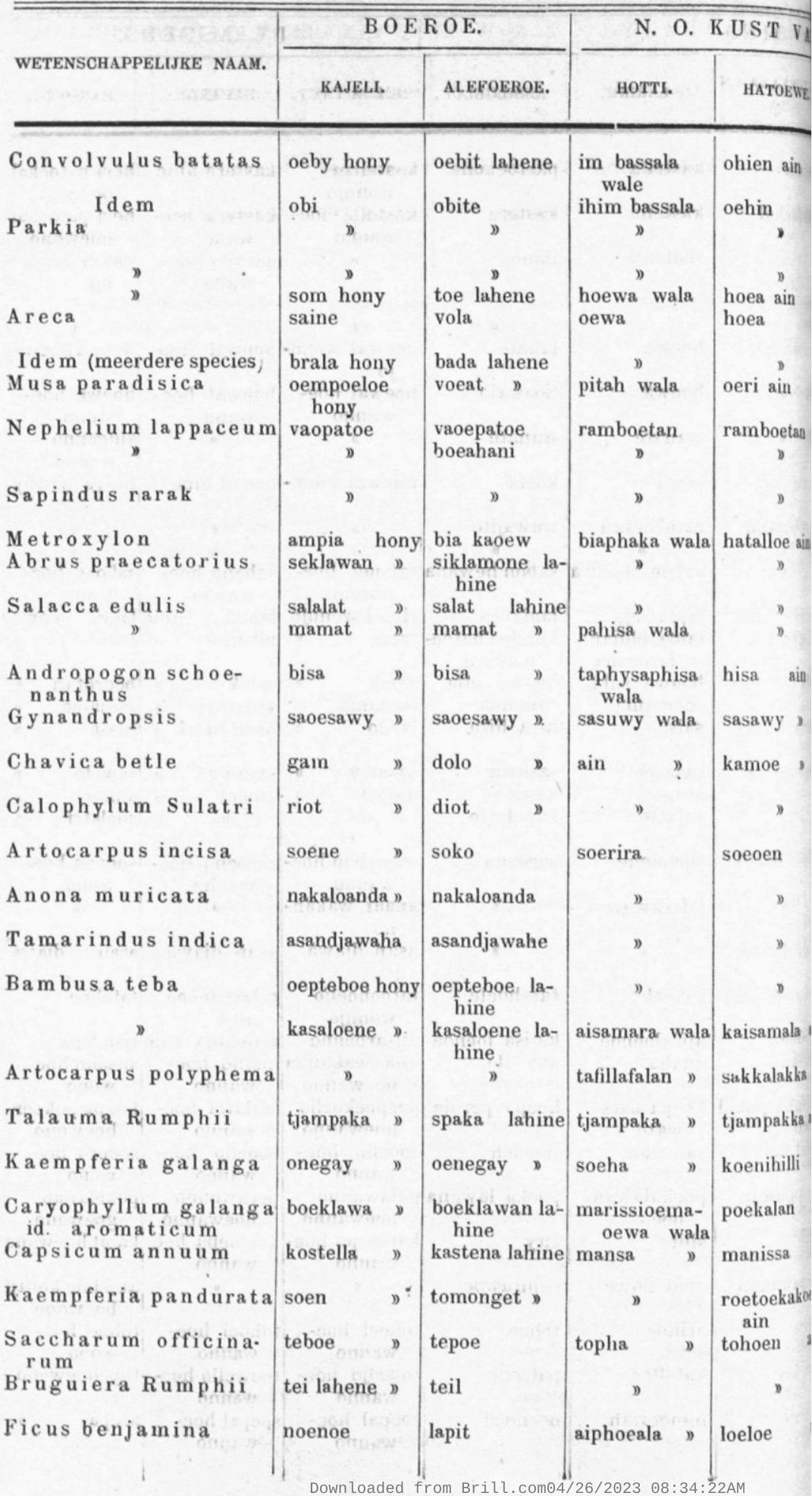




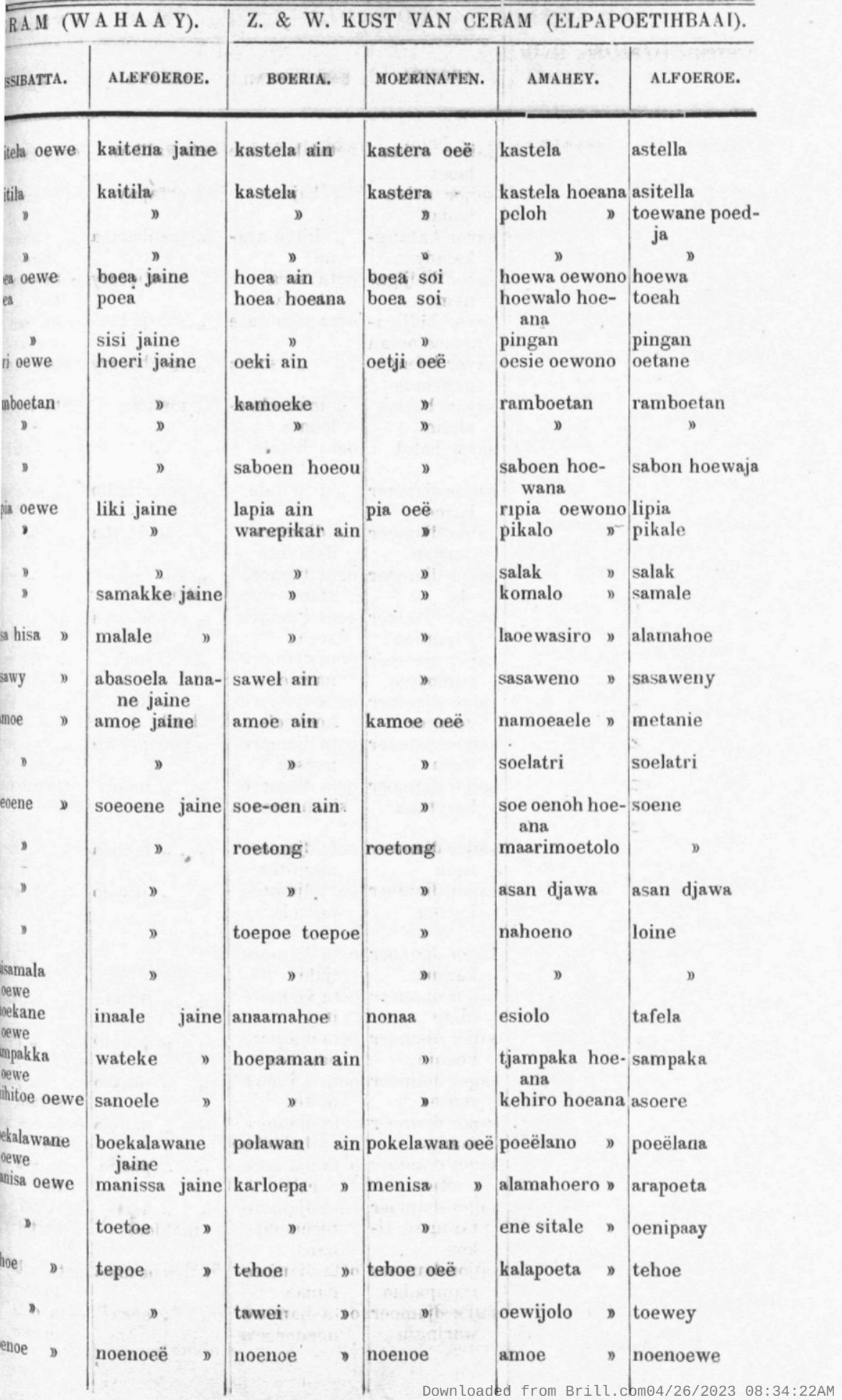




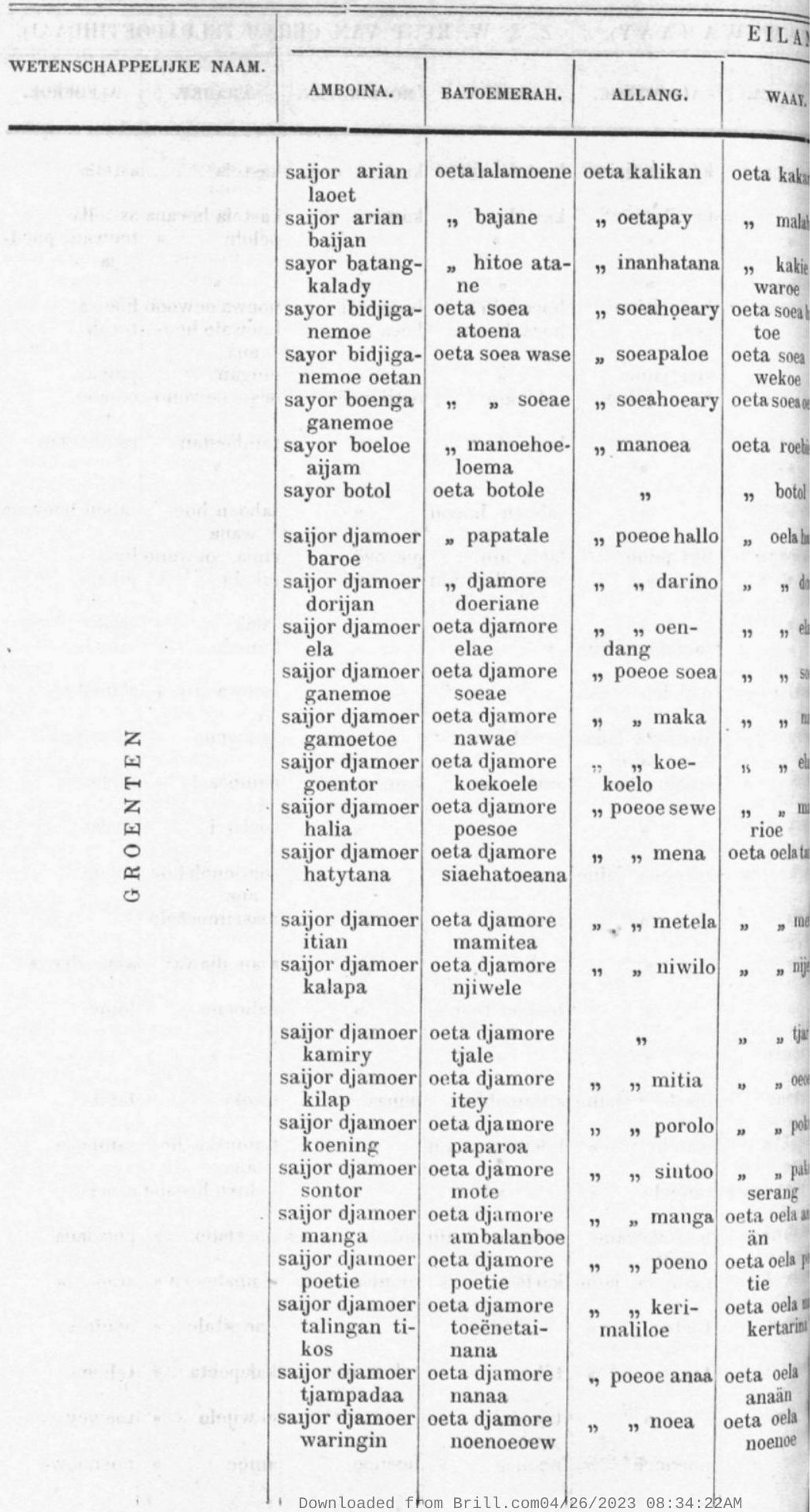


pain

apaoe-

apacts

at

"

"

"

$n$

botoI

soelehet

poetie

aloe

poetie

oeren

poetie ela

" oesa-

ea

poetie

awa

poetie

eoel

poetie

alia

poetie

ioe

poetie

peten

poetie

iwel

poetie ijal

, itit

kirie

mote

am-

alan

poetie

oetie

poetie

okietarima oeta poehoe

teloemaraloe

oeta poehoe

anaha

oeta poehoe poenoenoenoewa
B OINA.

ELAMA EN

HILA.

LARIKE.

NOESA LAUT.

SAPAROEA.

IIAROEKOE.

ULIA S S E R S.
ASSILOELOE.

oeta oeroea- oeta arienno ranno " lahoete oetan maia- $\begin{gathered}\text { oeta moela- } \\ \text { poete }\end{gathered} \quad$ " malahoe-

" kakilloe oetan topaale- oeta inanno oeta inan wahatanjo patai haloeny roete

oeta soea hina oeta tasoeaji oetan wisele oeta oeta soeal oeta soea hahoewan

w wasalolo oeta soea apala

" soewa

"koeta soea

$"$

hoeanno toey

oeta wekoel oeta weko hahoeanno toey

"parama- oeta oeta soea oeta soea oe-

leo ine koepanno oetty

" manoehin

"

oeta manoe- oeta manoe-

hoeroenno hoeroenno

$"$ poetie "

oeta botal oeta bitjibotol

"pahoe ha- oetane rape " oela hallo " oeraharoe loewe roemoe

oeta pahoe

poetoeren

oeta pahoe

erello

oeta pahoe

soeae

oeta pahoe

poehoemakalo

oeta pohoe

leloeno

oeta pahoe

poehoesewelo

oeta pahoe

mandjangan-

one atana

oeta pahoe

hoeloene

oeta pahoe poelo niwelo

oeta pahoe poehoejalo

oeta pahoe

poeitioew

oeta pahoe

koekoele

oeta pahoe

allahalo

oeta pahoe

poepoe japalo

oeta pahoe

laine

oeta pahoe

mamalotenana

oeta pahoe

nanah

oeta pahoe

poetienoenoe oetane doerian,$"$ toe,$"$ toe-

"wella oeta oela ela oeta oera ela

" wara- , , oeta , " dja-

male soeal moeta soea

oetane lapoeë oeta oelanawal oeta oera nawa

"talaei " " kiki- ", "sehi

" " "oeme- " " oeme

hoetoewanno

" niwile oeta oela me- ", "me-

tenno tenno

" ilahe oeta oela noe- oeta oera niollo mello

" oesilane oeta oela jallo oeta oera ijallo

"kokoroele "., itali- " , itallo

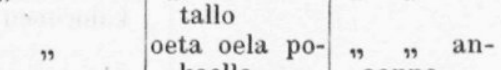

"papalam oeta oela ma- oeta oera moot

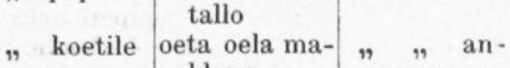

blang aanno

" marile- oeta oela poei- oeta oera poeta

tininaai tillo

oetane inaale oeta oela moe- " " ikailahal tir- "kalo nanno

"noenoeë oeta oela ana- oeta oera waallo kano oeta oela soe- oeta oera soepal paa "sepi $\begin{gathered}\text { natol } \\ \text { oeta oela sehil }, \quad \text { "sehi }\end{gathered}$ 


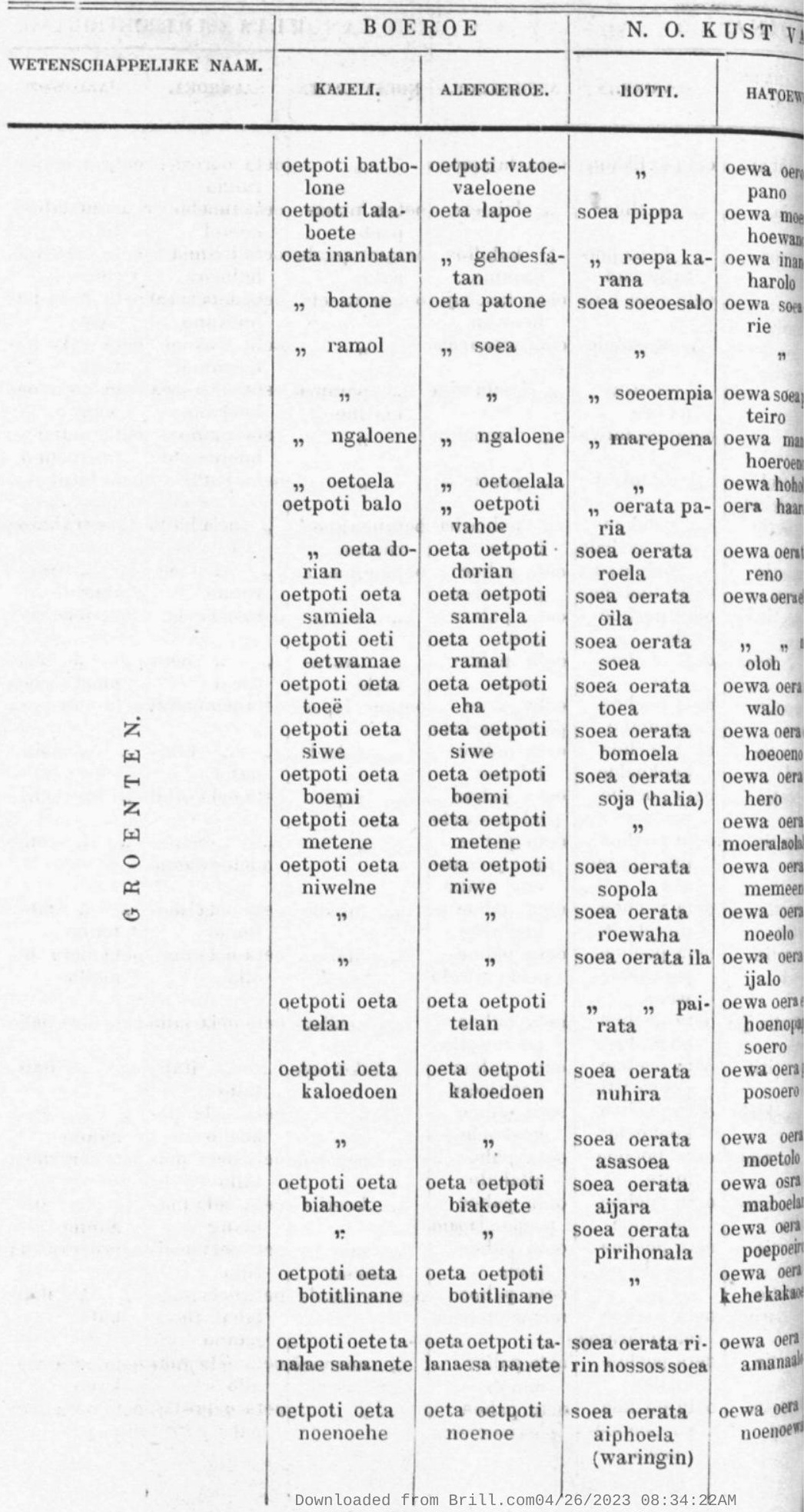




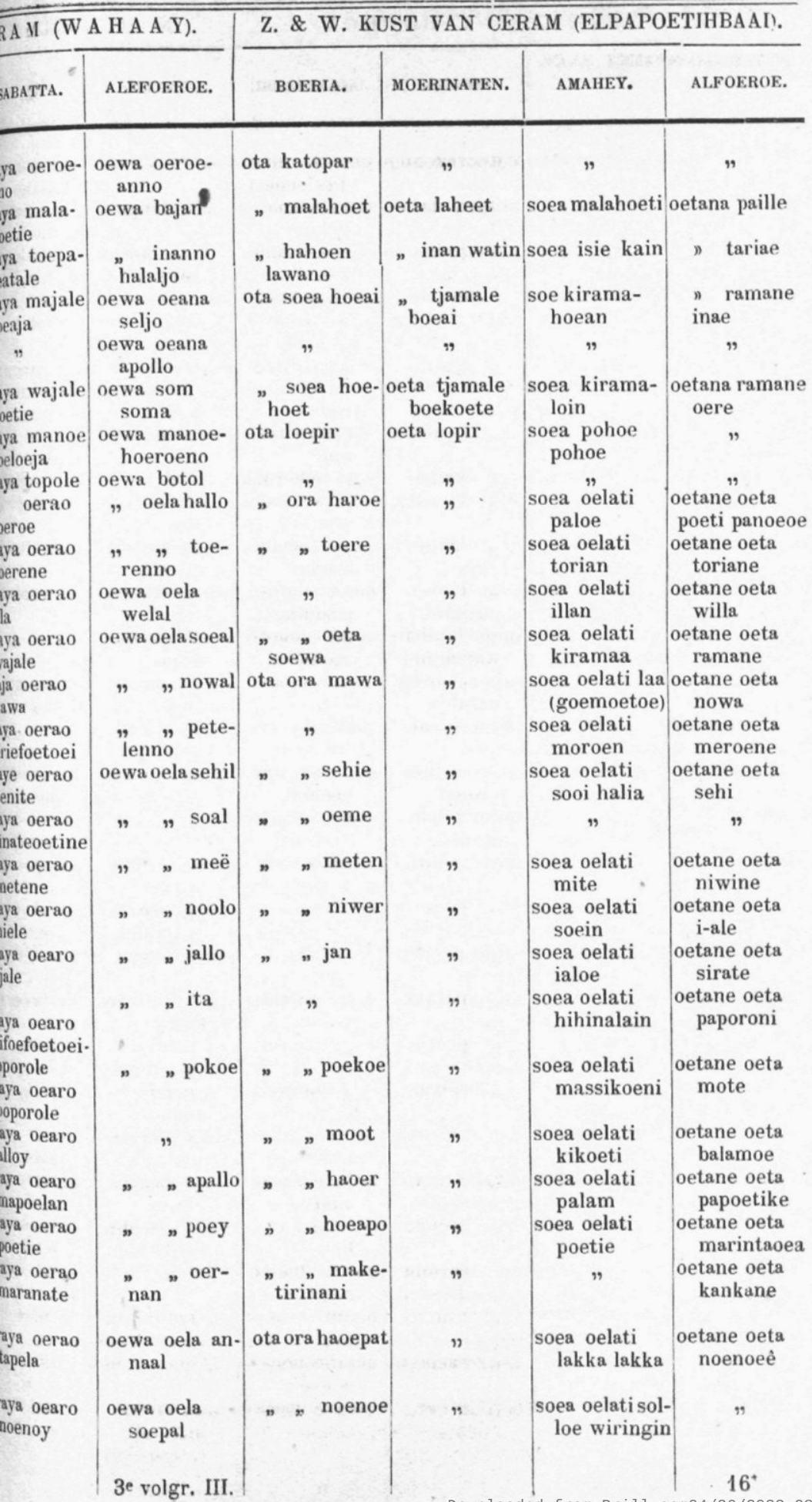




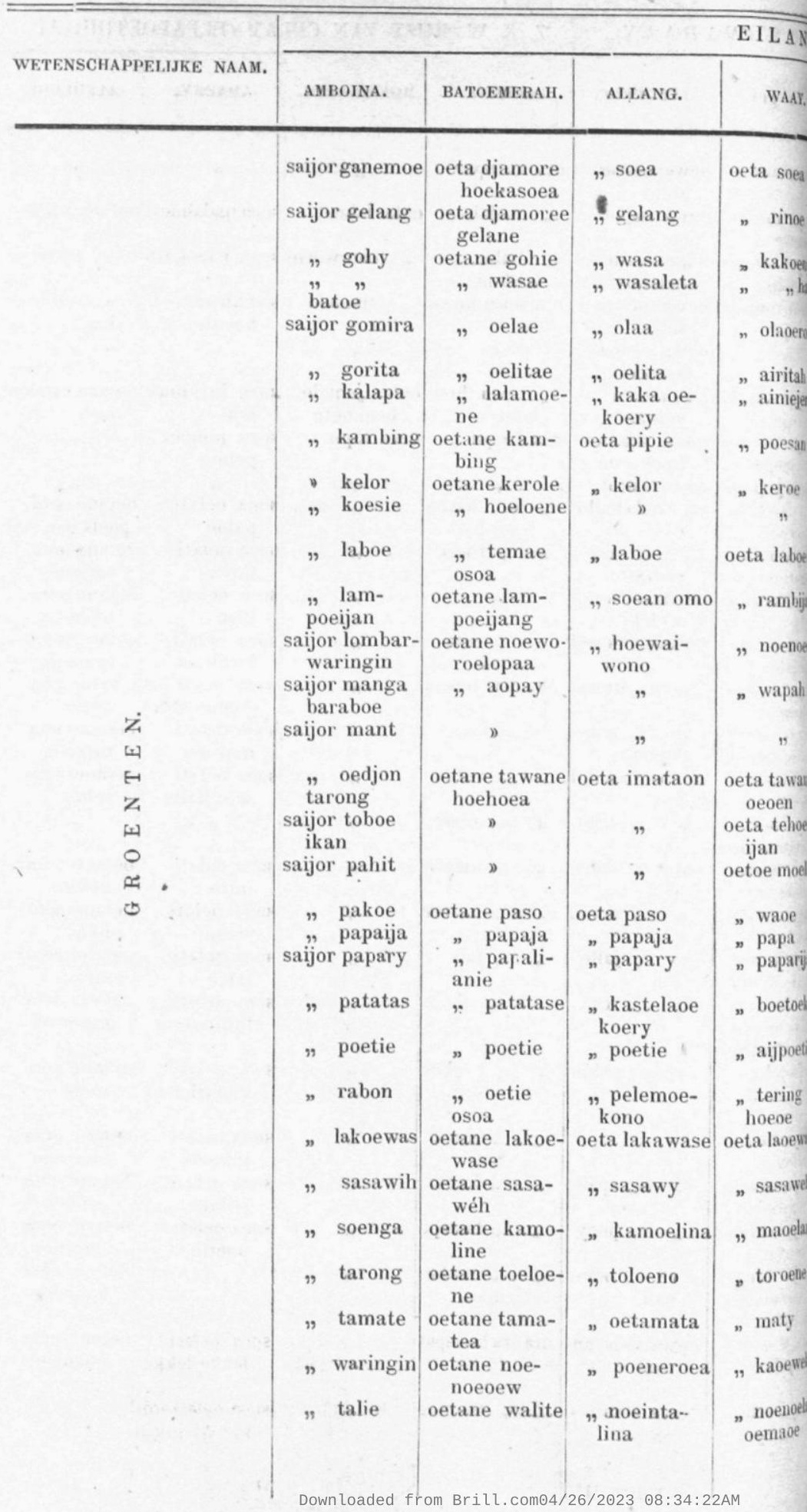




\begin{tabular}{|c|c|c|c|c|c|}
\hline \multicolumn{3}{|c|}{ IBO I N A. } & \multicolumn{3}{|c|}{ UL I A S S E R S. } \\
\hline $\begin{array}{l}\text { ELAMA EN } \\
\text { HILA. }\end{array}$ & LARIKE. & ASSILOELOE. & NOESA LAUT. & SAPAROEA. & HAROEKOE. \\
\hline
\end{tabular}

Isoera

noroet

ineoe

a wasa

a wasa ha-

be

$"$

$"$

niwel

pipie

keroe

,

a laboera-

poe

ta makalie

noenoe-

oely

ta ambala-

raboe

"

ta tawan

teloe

ta tehoe

oeijan

ta noenoe

oelie

ta paso

ta papae

paporian

partoekal oeta kastella

$$
\text { ai }
$$
aipoetilan

ta laoewase

sasaweh

maoelanit

toeroeng

tamate

noenoe toeha oeta oelah oeta pipie oeta lopo

v lampoeana

) lapomiwa

" ijapaloe

n mata

» tawanoe ikan oso

) paso

) papaja

) paparianoe

) poetite

๖ telinose

). siwaho

n koemoelenin oeta taloeno

) mate

n poenoenoewa oeta walita oeta oetasoewa oeta soewalo " kelannoe oeta koroto oeta wasa „ wasalo » soeha ha- "soea ha-

" oelita
noerwelloe

kerollo
" teloe ma-
rillo

v toboe oeta oerealoe

\section{wahie} toeinjo oeta allawoe

n oeloetoe

v poetie noenoe oeta pipija

) kerolle , mamalotinano oeta lapoe

) siwa oelo

n noehoenoehoelopa ota tapoetie

m) matello

v tawane

n toboe ikan

) tanamo oeta pawoeloe oewa moeoeno oeta wasol oeta waoew

"pajalaoene " papanno " papaanno oeta papae

" papariane "paparianno "paparianne " paparipo-

n oehie

) aipoeloe

) kastella

D) teline hosaa

oeta kakoease paoea

) sasawelloe oeta sasawe

v koemoelinoe oeta toeroeno

owa patamale oeta oeta soeal oeta oera soea

\begin{tabular}{|c|c|c|}
\hline$"$ & "gelang & toe $"$ rinoe- \\
\hline wasal & v wasal & oeta wasal \\
\hline $\begin{array}{l}\text { wasa } \\
\text { waljo }\end{array}$ & $\begin{array}{l}\text { "wasal } \\
\text { hatoel }\end{array}$ & ) wasahoeta \\
\hline
\end{tabular}

oewa moesoe- oeta soeoetol „ alloroe tal

") oerital ritalaoey

„ pipil „ korabate " poesanpan

" kenello " kerello „ kerel

» moeoenno " makello " makello

» lapoe „ rapoe laboe

n sei oepan- "sehioe- " tripitoe-

\begin{tabular}{l|l|l} 
no & panno
\end{tabular}

oewa lopail oeta soepal oeta toeello

soepal oewa apal

) tehoe

hoetoeil raoey

oeta tyapoe- oeta tehoeijan oeno

oehoey

oeta tehoeijan

maato

” wapaa

1) mooewa-

oey

n kastera ceta partoekal oehoery

n poetil

n poetil

oeta poetiijra-

1) rabon

b tomoroello oeij

oeta tering oehoery

n laoewasil oeta laoewasil oeta laoewasie ahoetil

» sasaweh oeta sasaweh „ sawelo

" poelanno " oepoe- " hahoew

n mata

1) poetie

noenoe oeta walete
" toeronuo oeta toeroen- "toroenno

\begin{tabular}{l|c|c|c} 
"toeronuo oeta toeroen- & "toroenno \\
no & tamate & oeta tamata & " tamate \\
"soepal & "soepal & $\begin{array}{l}\text { "noenoe- } \\
\text { oew }\end{array}$
\end{tabular}

n noemoeol " noenoelo oeta toeelo 


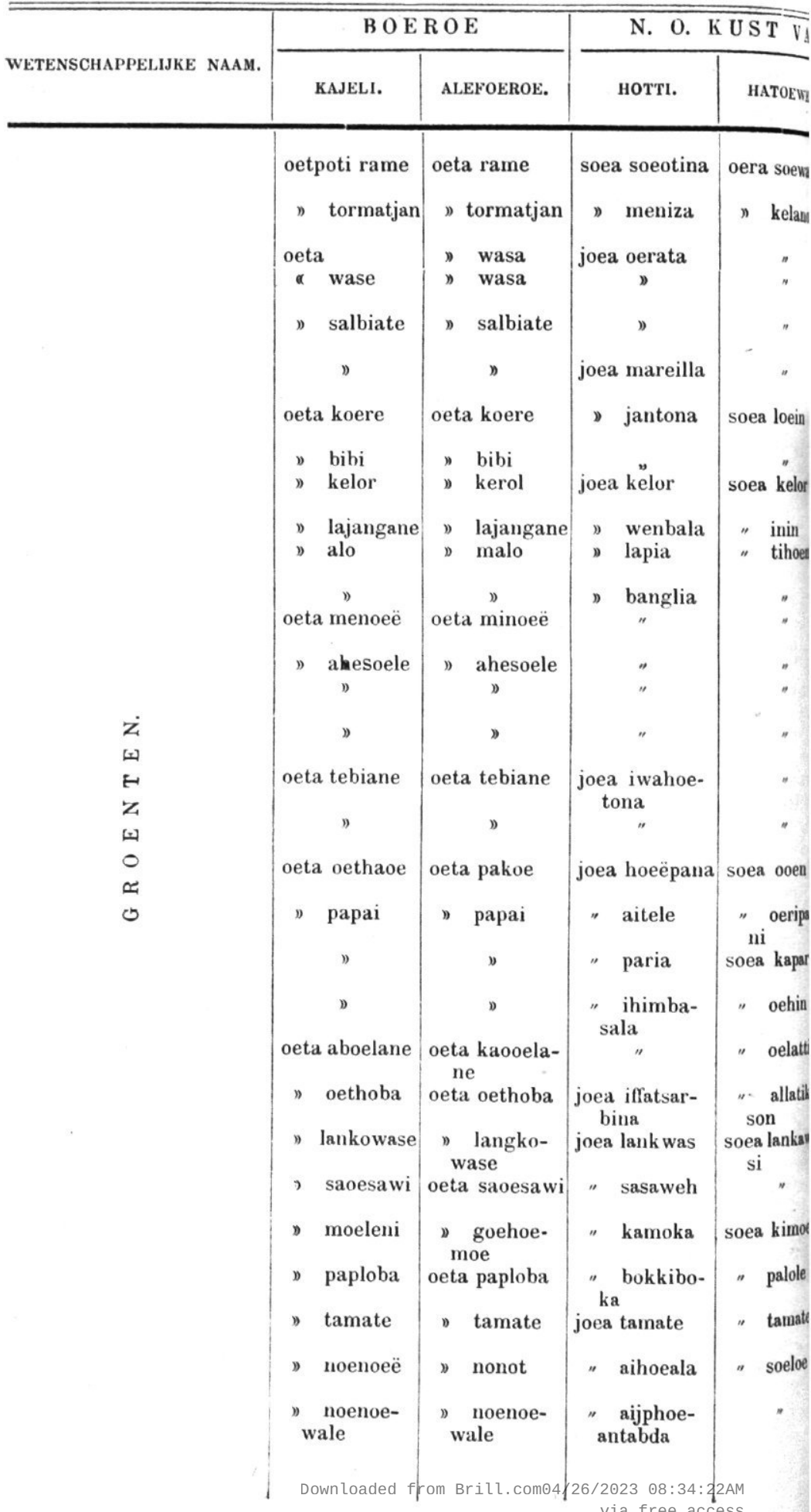


RA M (W A H A A Y).

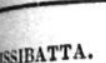

ALEFOEROE.

ISIBATTA.

Z. \& W. KUST VAN CERAM (ELPAPOETIHBAAI).

BOERIA. MOERINATEN.

AMAHEY.

ALFOEROE.

iijosao

rajale

raija topce-

.

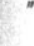

tane niwine

,

tane kelor

"

ne laboe

,

,

,

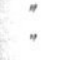

.

tane waoe

kaitila

paripari

kaitila

tane lakoe-

rassi

"

tane kamonine

tane palola

tainate

noenoeë oewa soeal

y gelang

"

$$
\text { " }
$$

"

$$
\text { " }
$$

oetane niwele

" kelore

" kapoele

n

"

"

$"$

"

$"$

"

" haiwano oeta haoe

" kaitila " papaie

oeta haoe

" keoe keweli

" paparian oeta paparia

" kaitila

" kastella

" kastera

" aipoetie

" tering

tahoerie

" lakwas- toea lawasie sie

$$
\text { sie }
$$

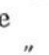

etane palola

" sawel

oeta sawel

oemelanit " oemerene

" toeroen

" toloen

" teling

osoo

,

西

" hahoen oeta hahoen-

oewa akaoero taraiea waasa

" haoeha- " waasa

oere patoe

oewa noenoe taraiea koesa

oloole koesuea

oewa oerialo taraiea oeria

n noeoloh niele

n) makolo mokole

»kerelo » kerele

D lapoero " " lapoe

" opanno " opano

๖ noenoe $"$ noe-

lopairo noedelopay

oewa loka palo taraiea leleah

" "

D tawano b tawane

oewa oehoe taraieamasite yane jane

oewa oedocro taraiea paoewe

" papaja " papah

b paparyane " papa-

" kastella taraiea asie-

tella

v aipoeiro taraiea aypoe.

tiejo

- ereno taraiea terine

hoero rifoey

oewa awasi taraiea lawase

oehoeiro toepoey

oewasasaweno taraiea sasa-

) poerono taraiea amoe-

lina

- oeroeno taraiea toe-

n tamate taraiea tamate

• noenoe- „ noenoey

wolo

oewa noenoe

olo olo

n ritiritie 


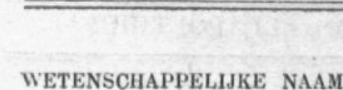

Bamboe worm

Buideldier

Bloedzuiger

Bijen

Casuaris

Civet kat

Duizendpoot

Geit

Hert

$\mathrm{K}$ ip

Krokodil

Muskiet

Schildpad (zee) id e m (land)

Sehorpioen

Slang (groote)

id. (gift)

Spin

Varken

Vledermuis

\section{.}

AMBoINa oelar bamboe koesoe lintah

niri

caswaris

toesah

kakie sariboe

kambing

roesah

ajam

boeaja

njamoe

tataroega laut id. maijmay goenting oelar bezar id. bisah

lawa lawa

babie

marsego

coecoe

goheba

kakatoea merah id. poetih

nija

atole nija elaka nija bisa

kondo itam

boerong kon do boedjang

boerong kondo poetie

boerong mata merah

kawene

manoe maneij oewa poetia boerong mara paty

boerong mara paty ejkor panajan

boerong maleo

id. maengolo

boerong oepoe

boerong pom bo biroe

boerong pom bo poetie

boerong radja oedang

boerong taon taon

boerong tjoei manoe tfoei ouw poeroe kone manoe mala poetia nittale manoe $\tan$ gonnge

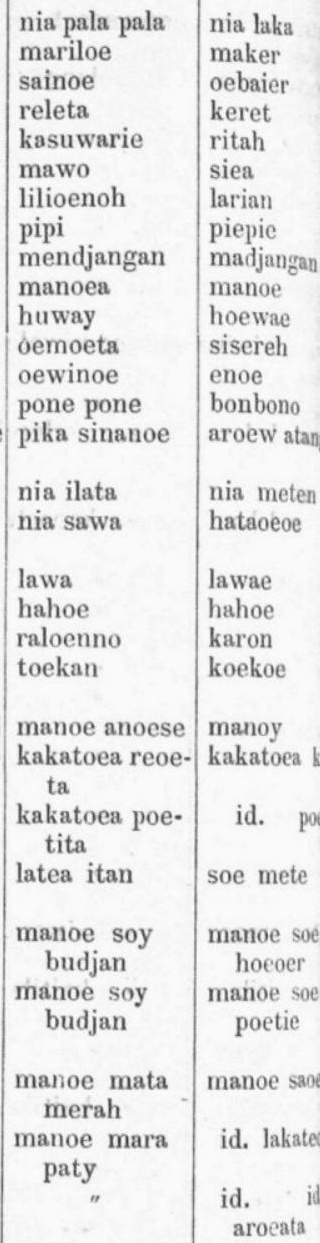

manoe lotea

manoe met

id. hia oe-

manoe mala manoe oepoe(19)

EILA

WAAY,

nia lak

oebaie

siea

piepie

nadjangan

hoewae

sisereh

enoe

bonbono

nia meten

lawae

hahoe

karon

" paigole

id. baik

" sokea

manoe sakoe manoe sho tioe apolo lala

manoe papaoe manoe aho poetila poetie manoe radja manoe sali oedan nie manoe kyopa manoe alla

" kroebita 


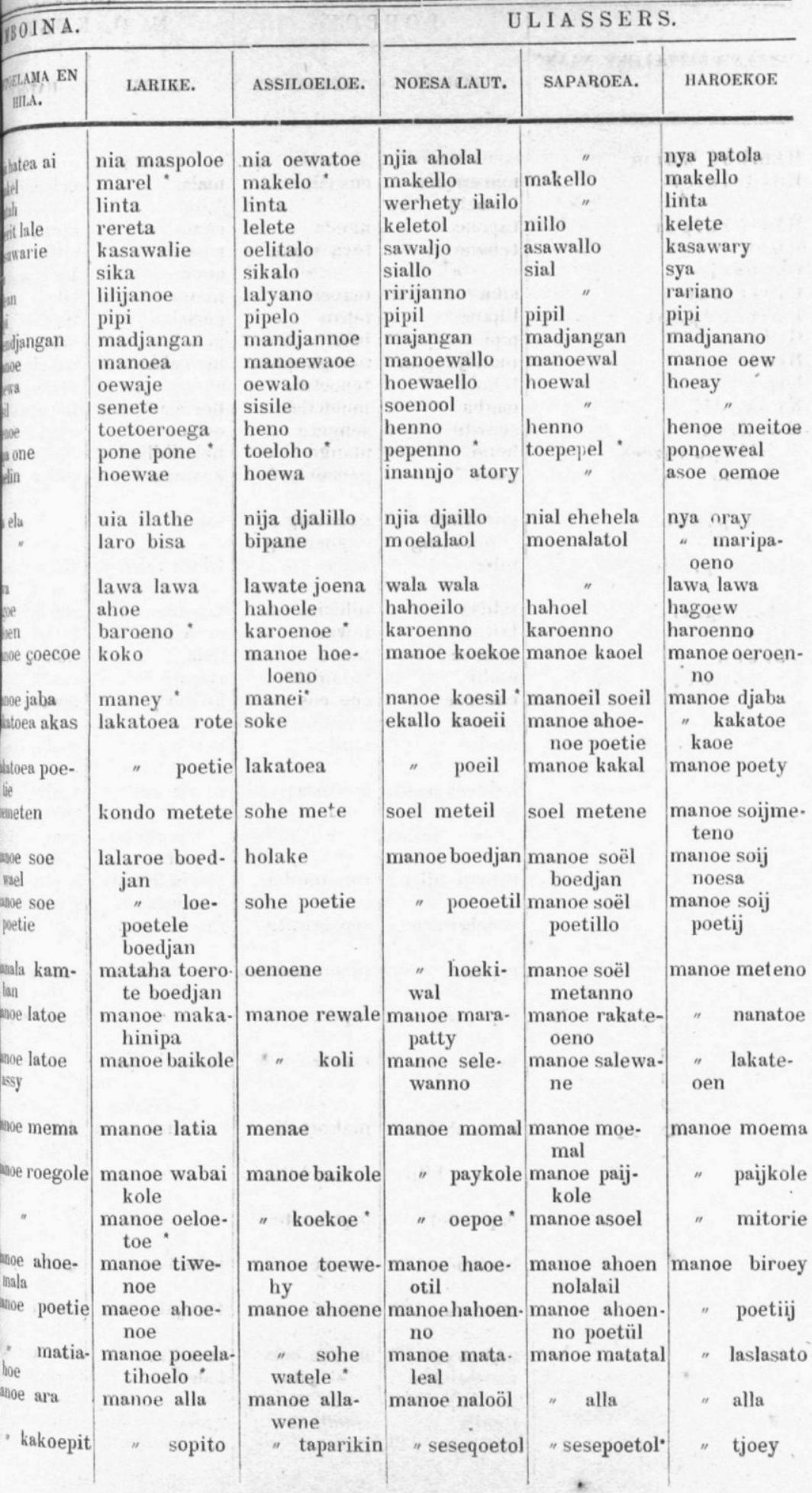




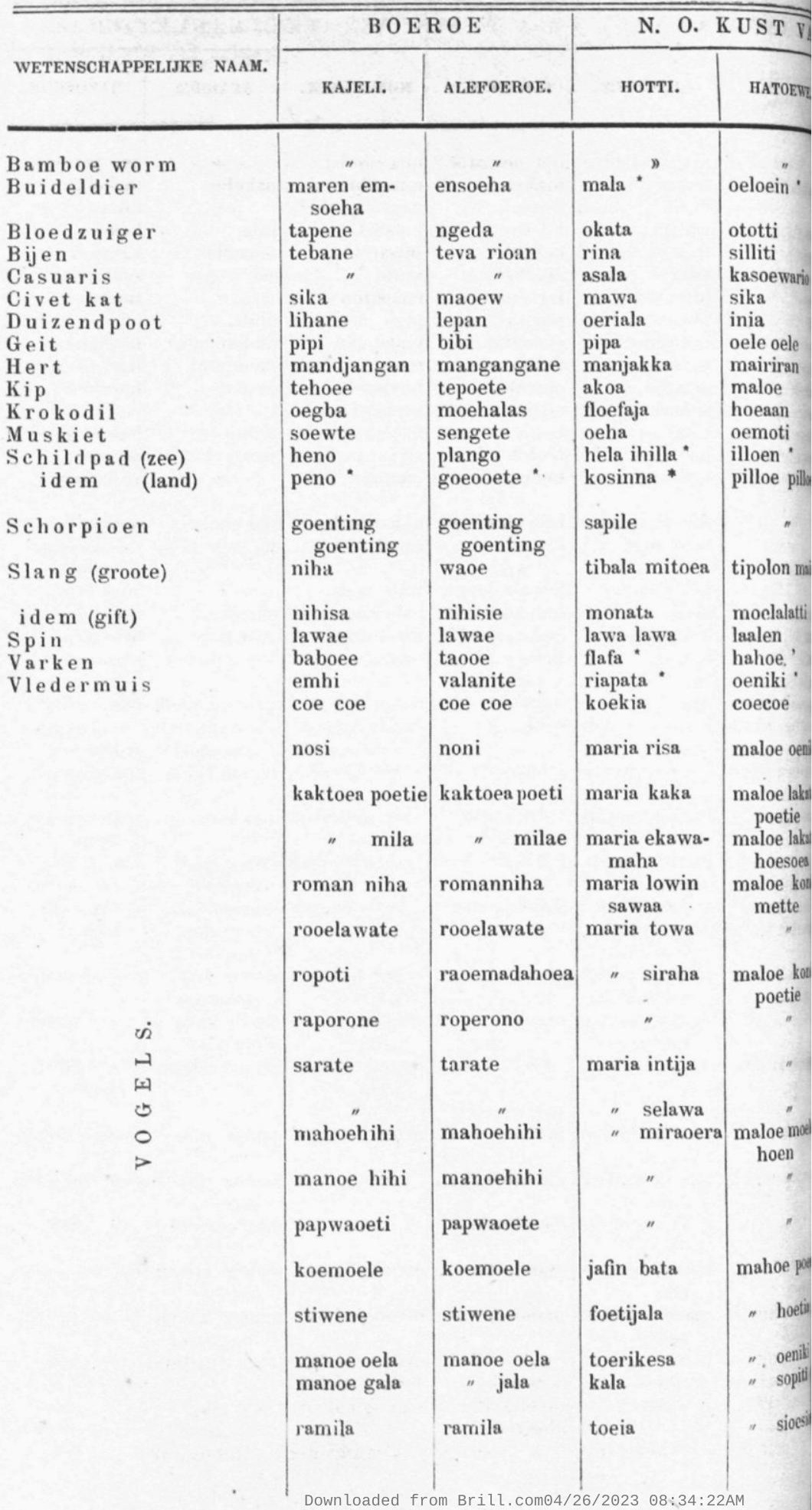




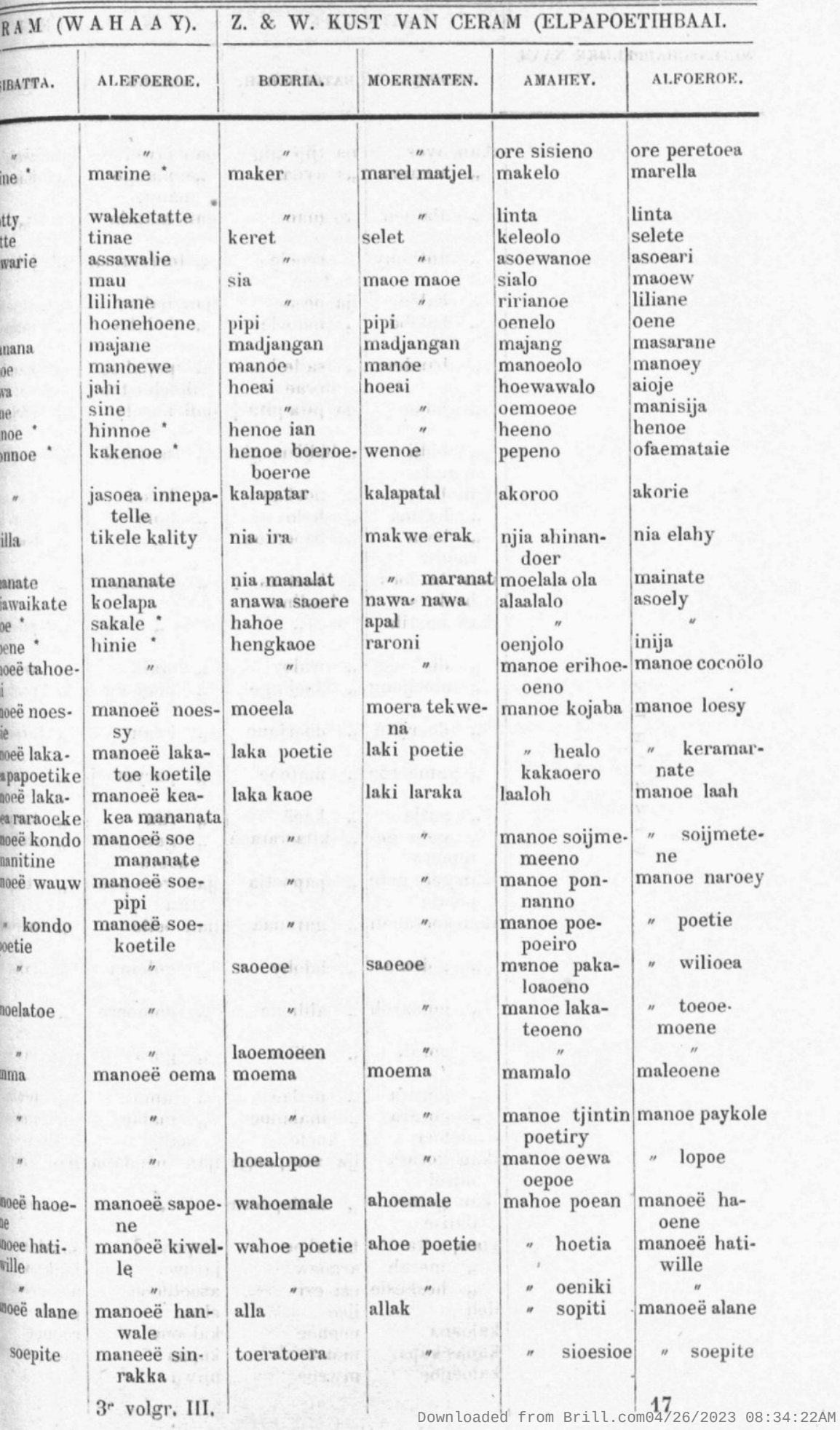




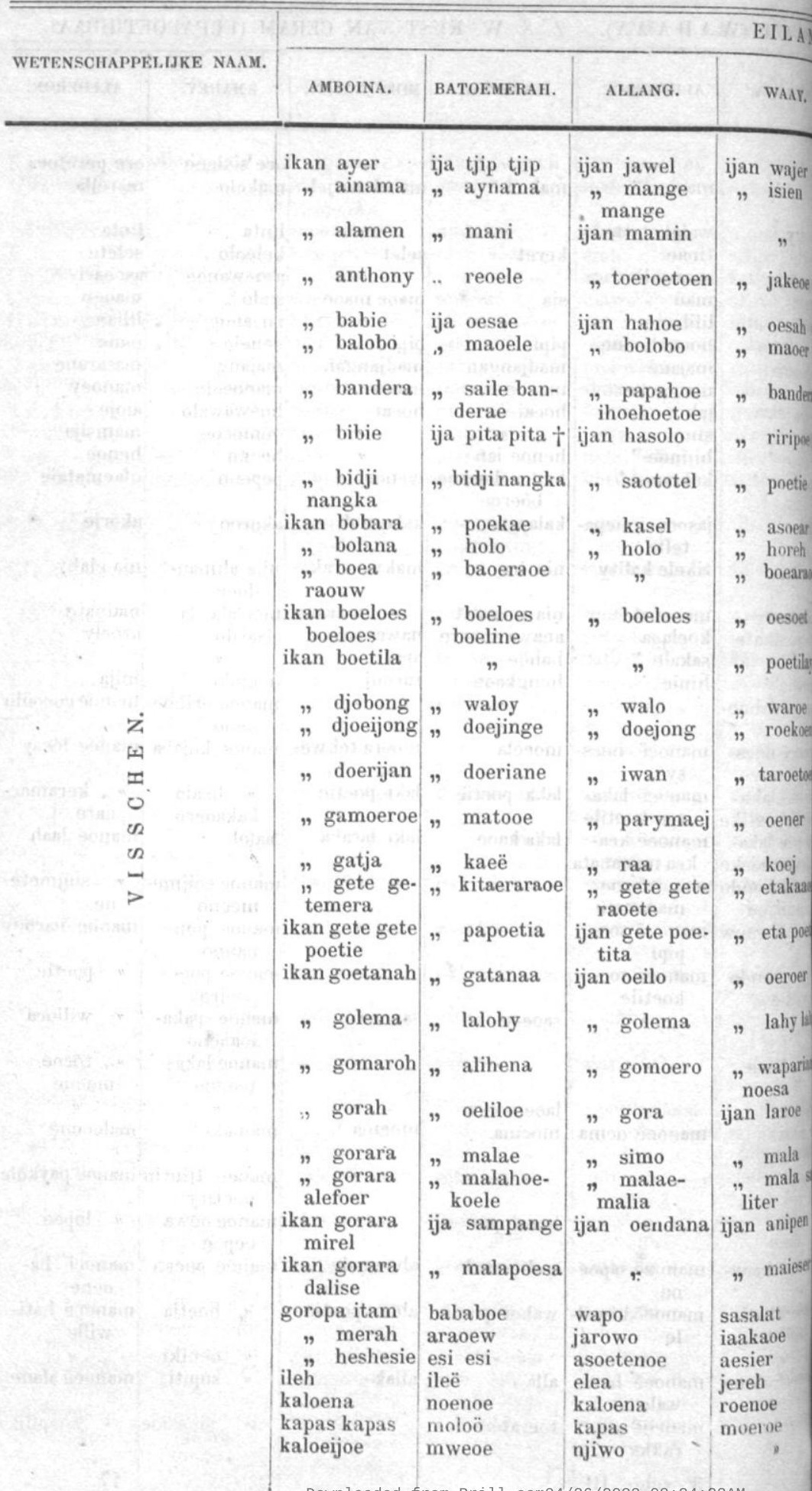




\section{BOINA.}

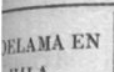

HILA.

LARIKE.
U L I A S S E R S.

ASSILOELOE.
SAPAROEA.

HAROEKOE.

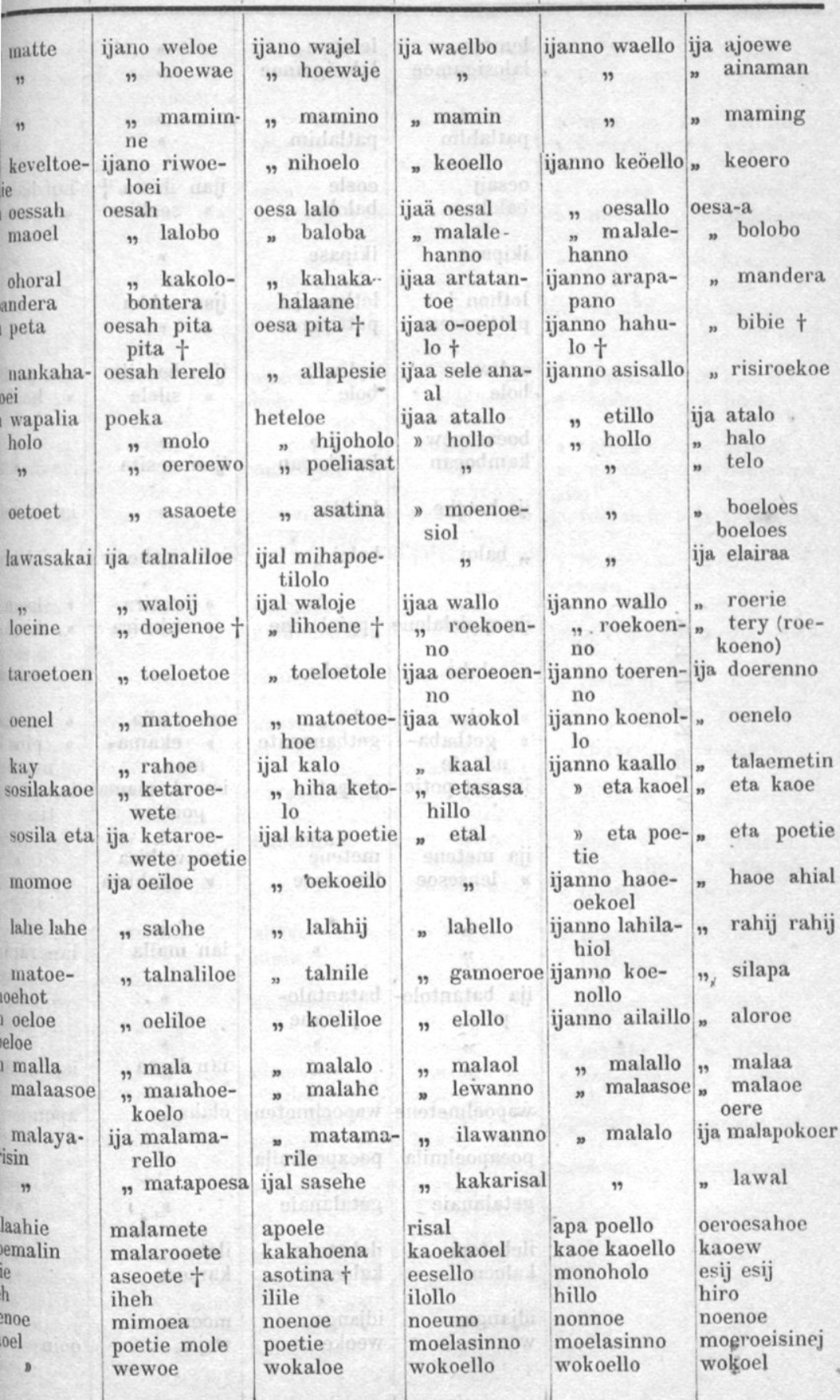




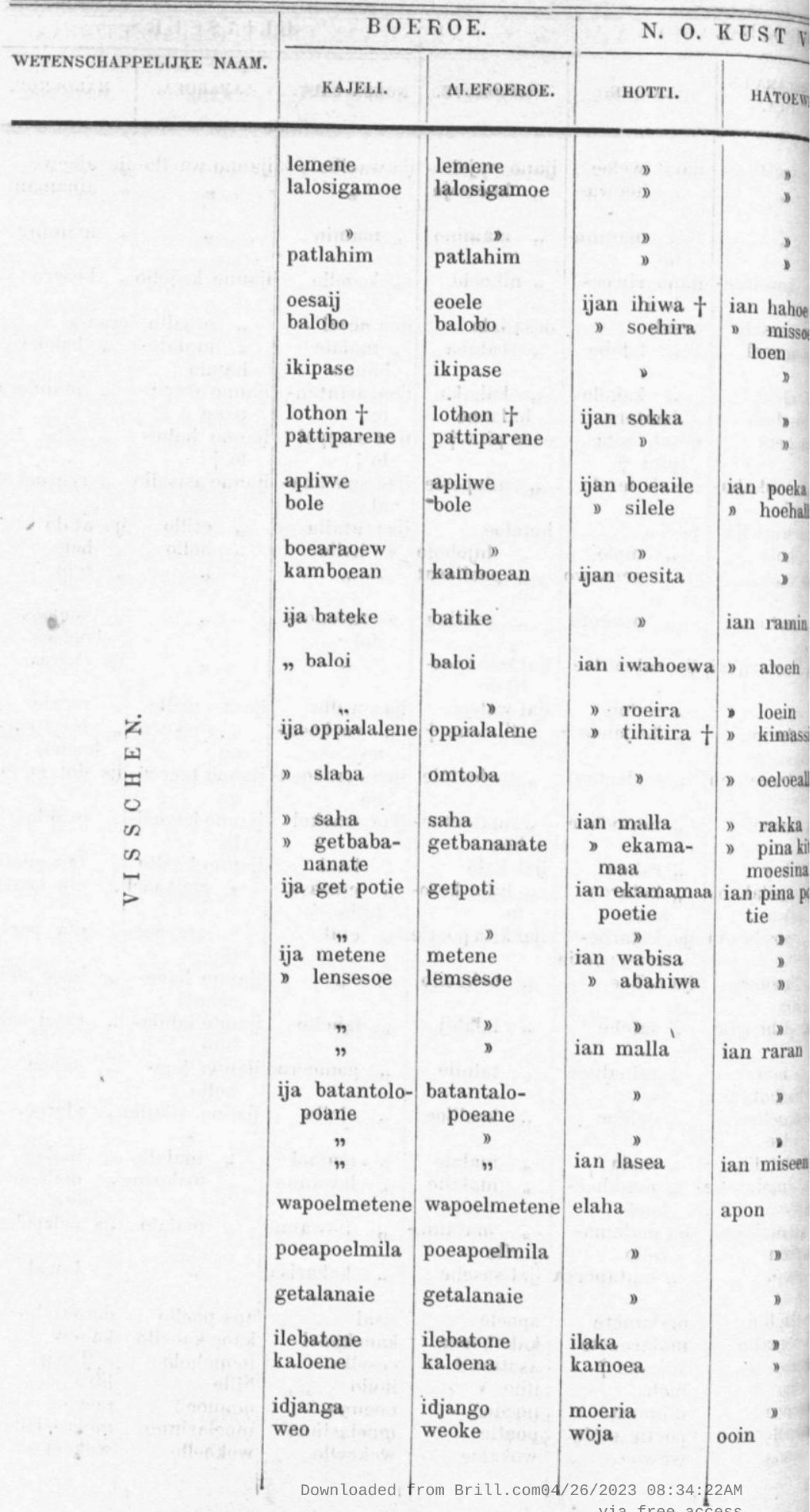




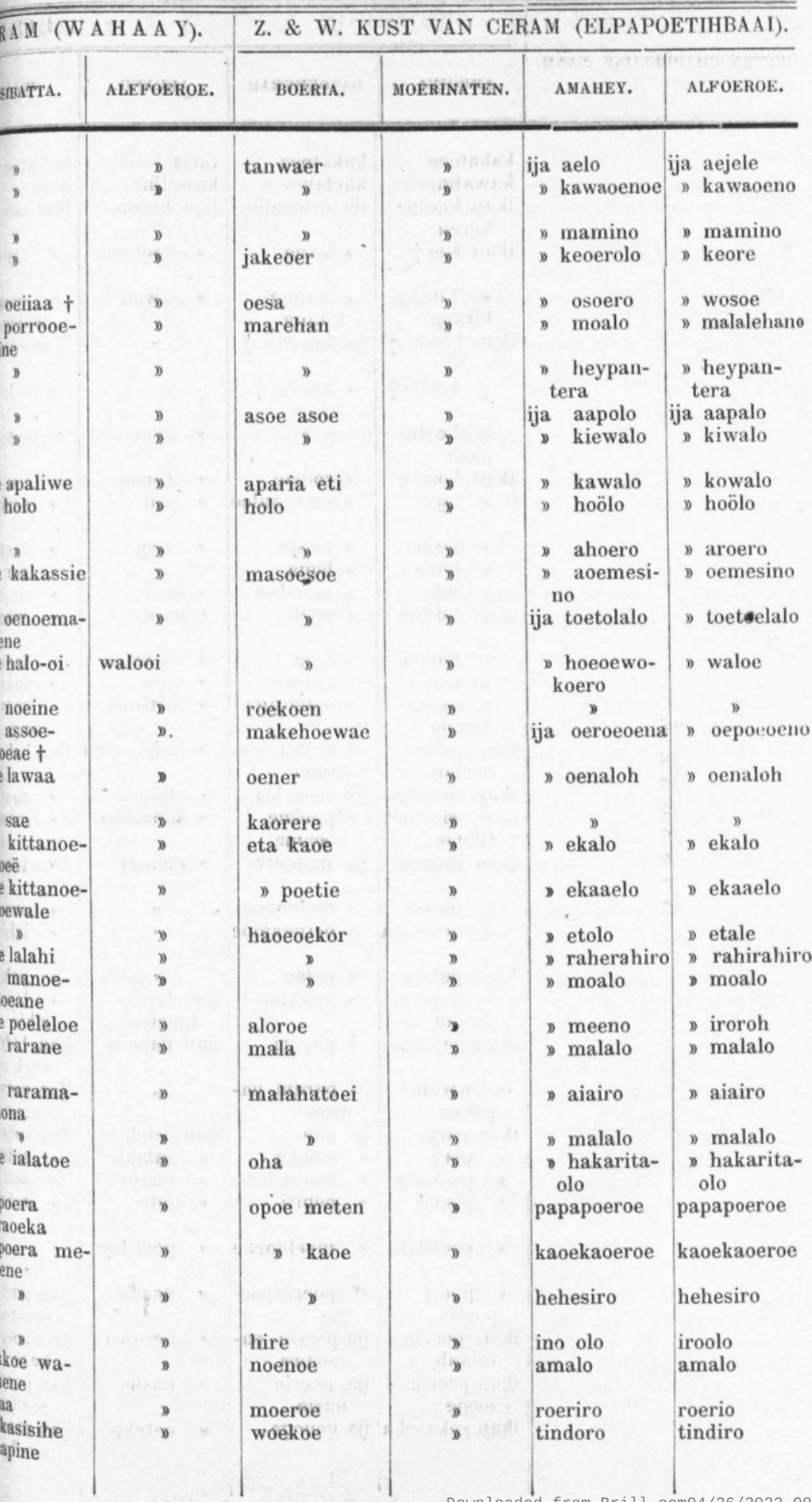




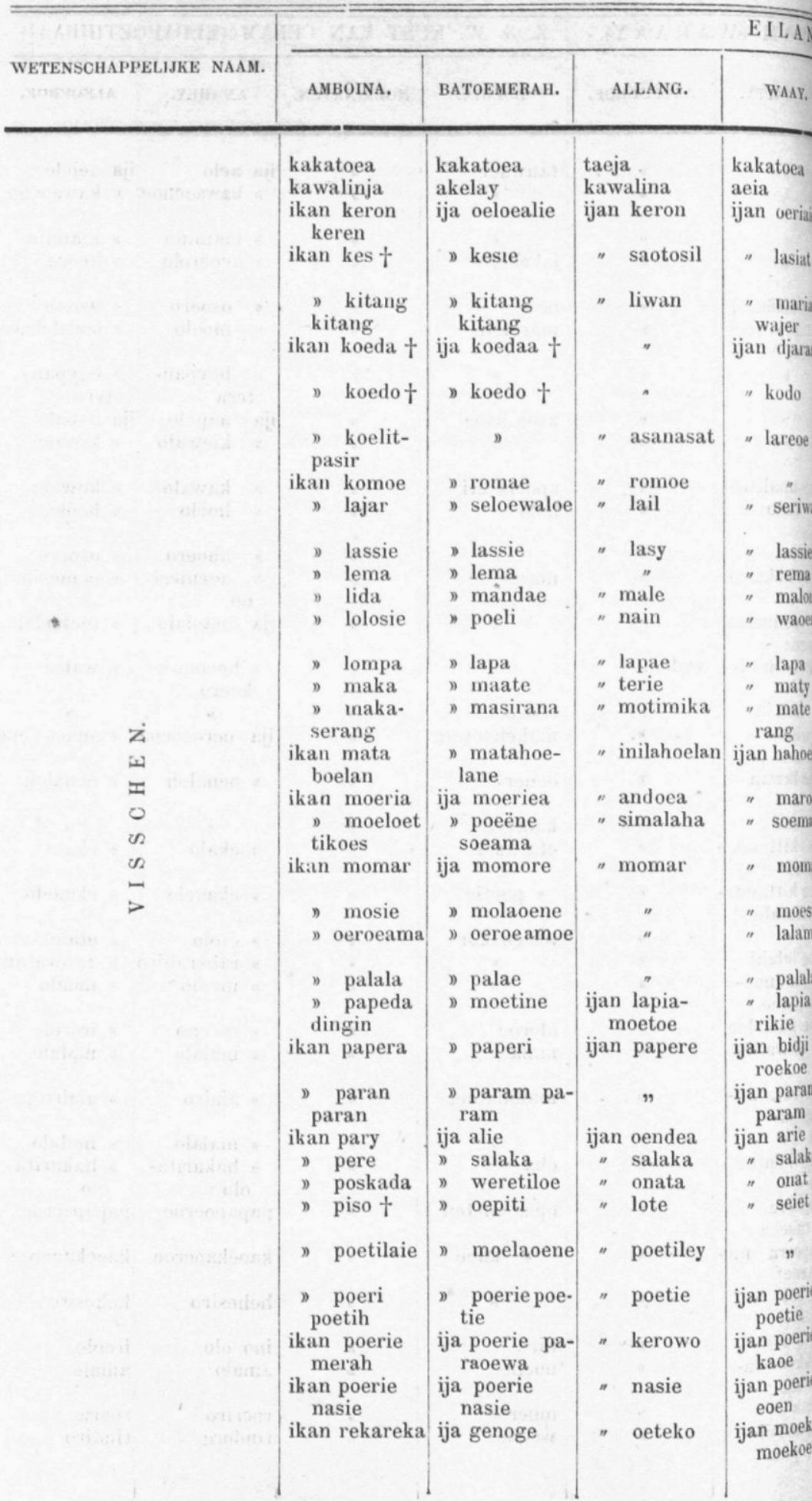




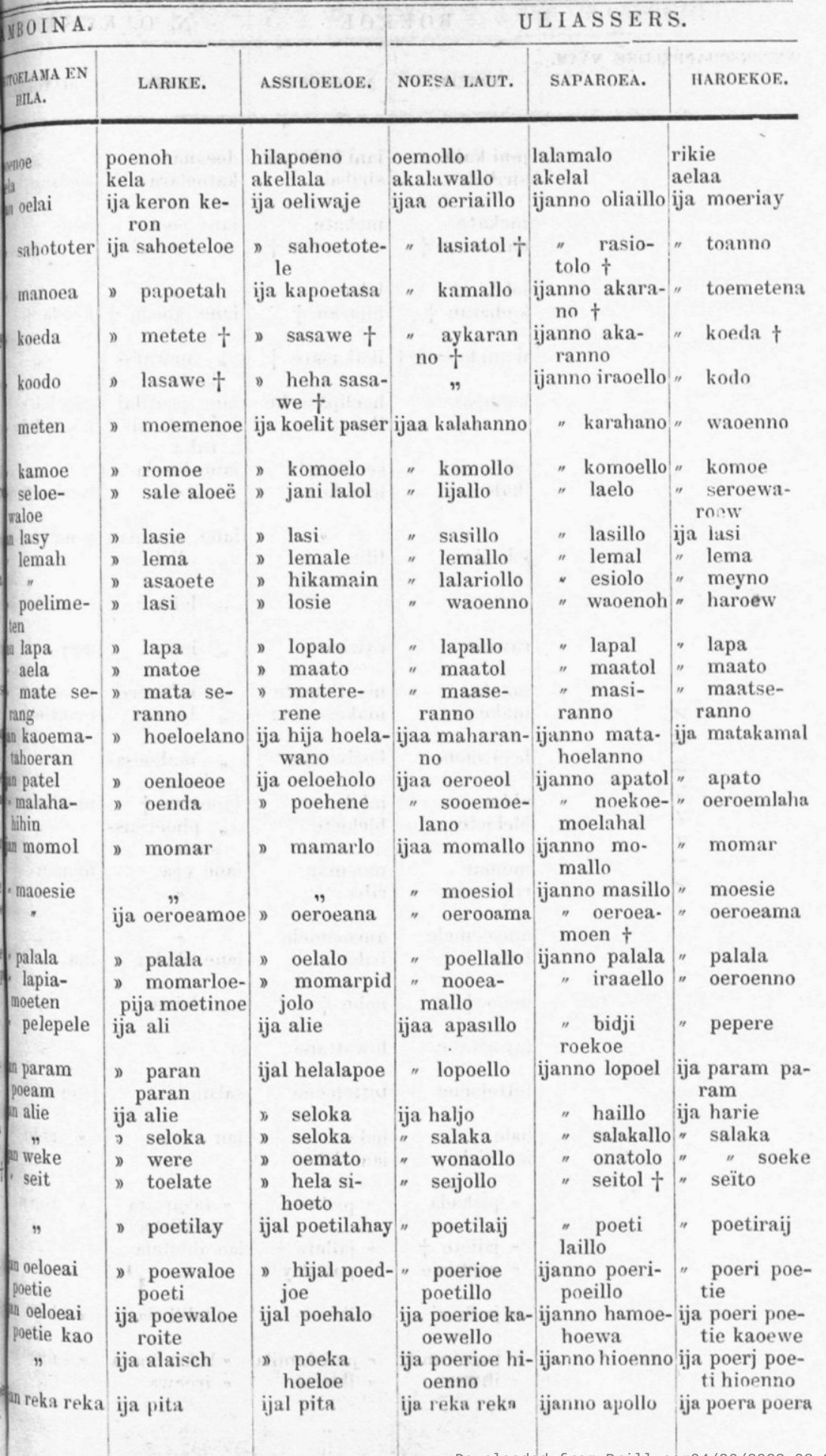




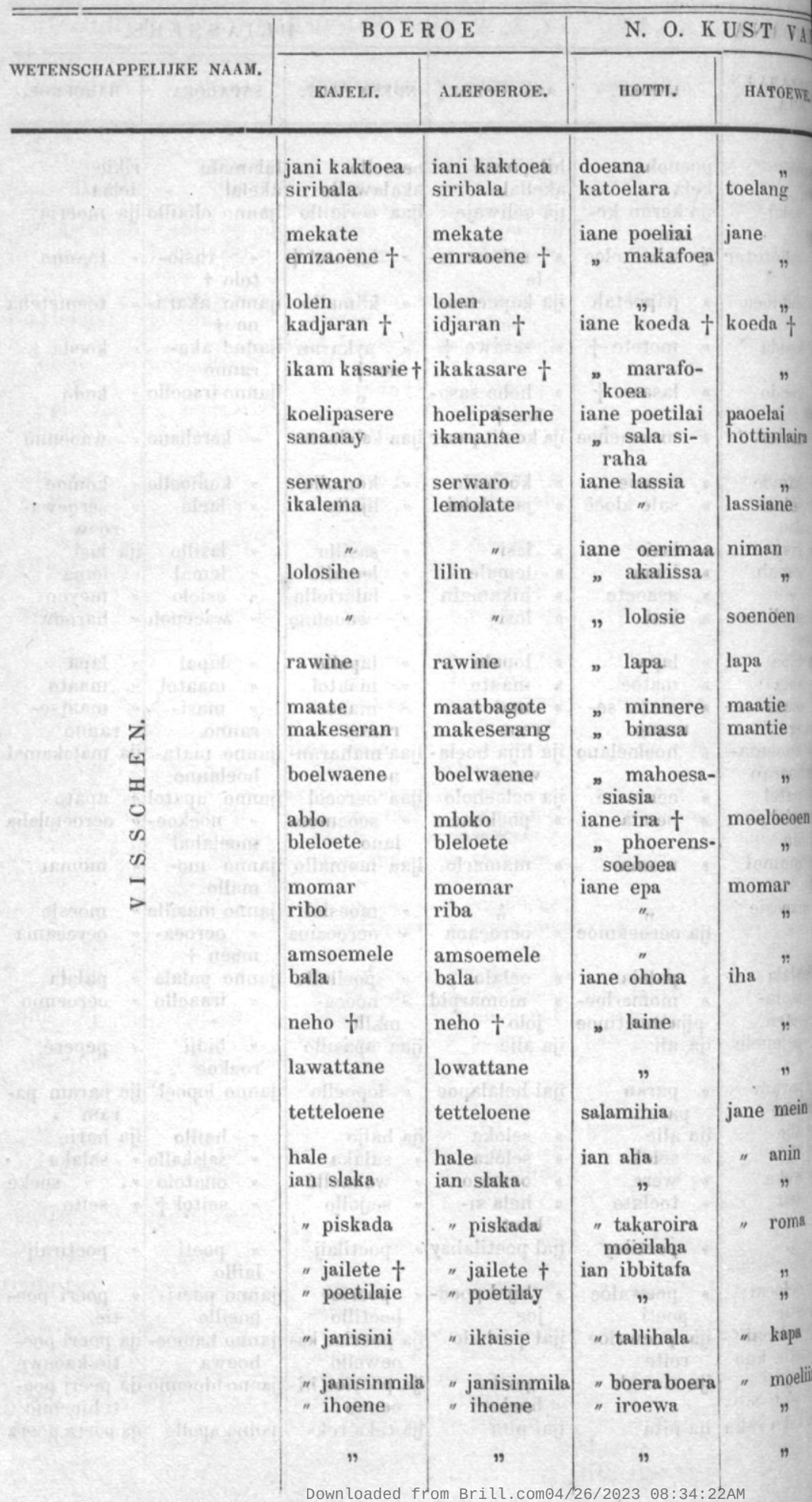




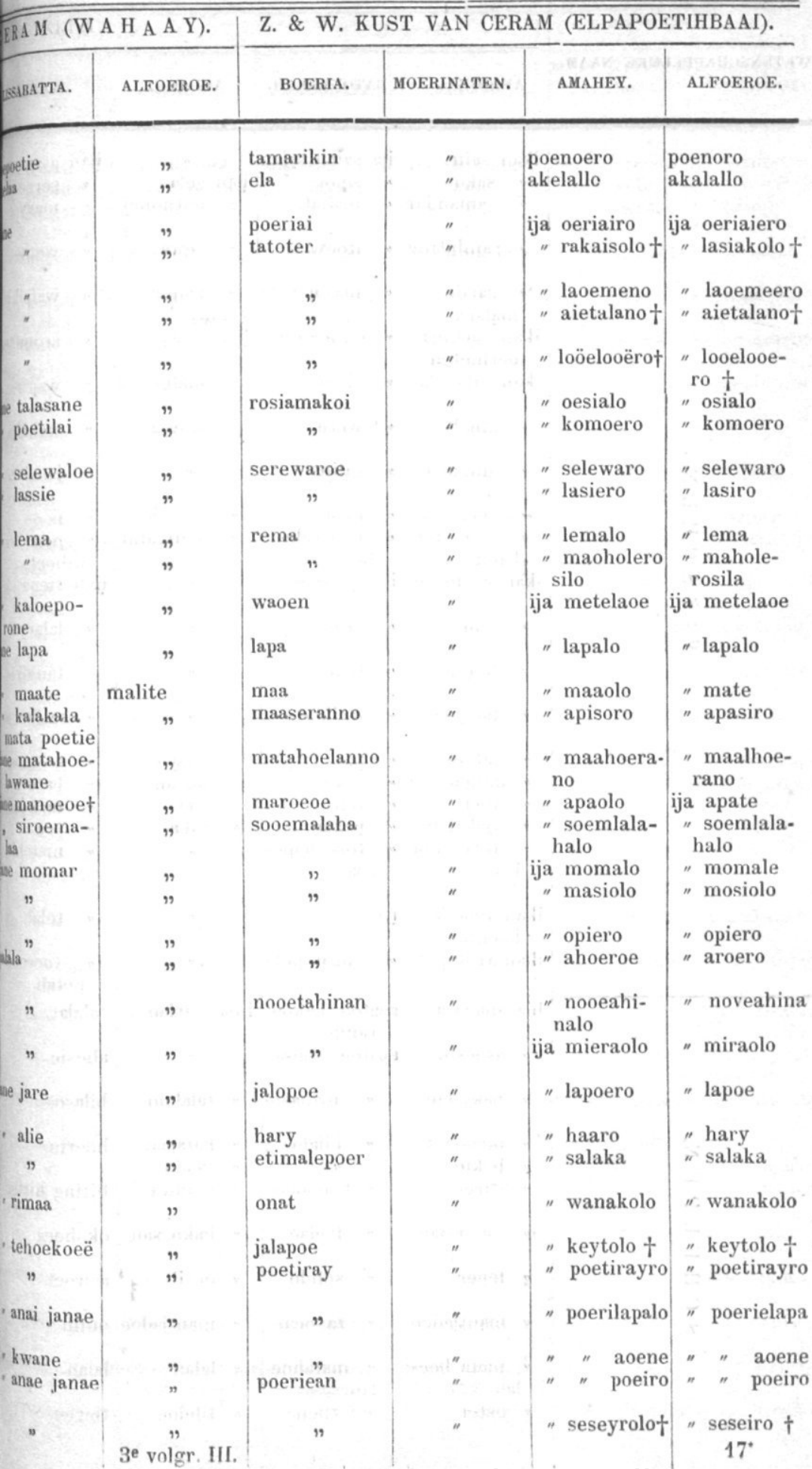




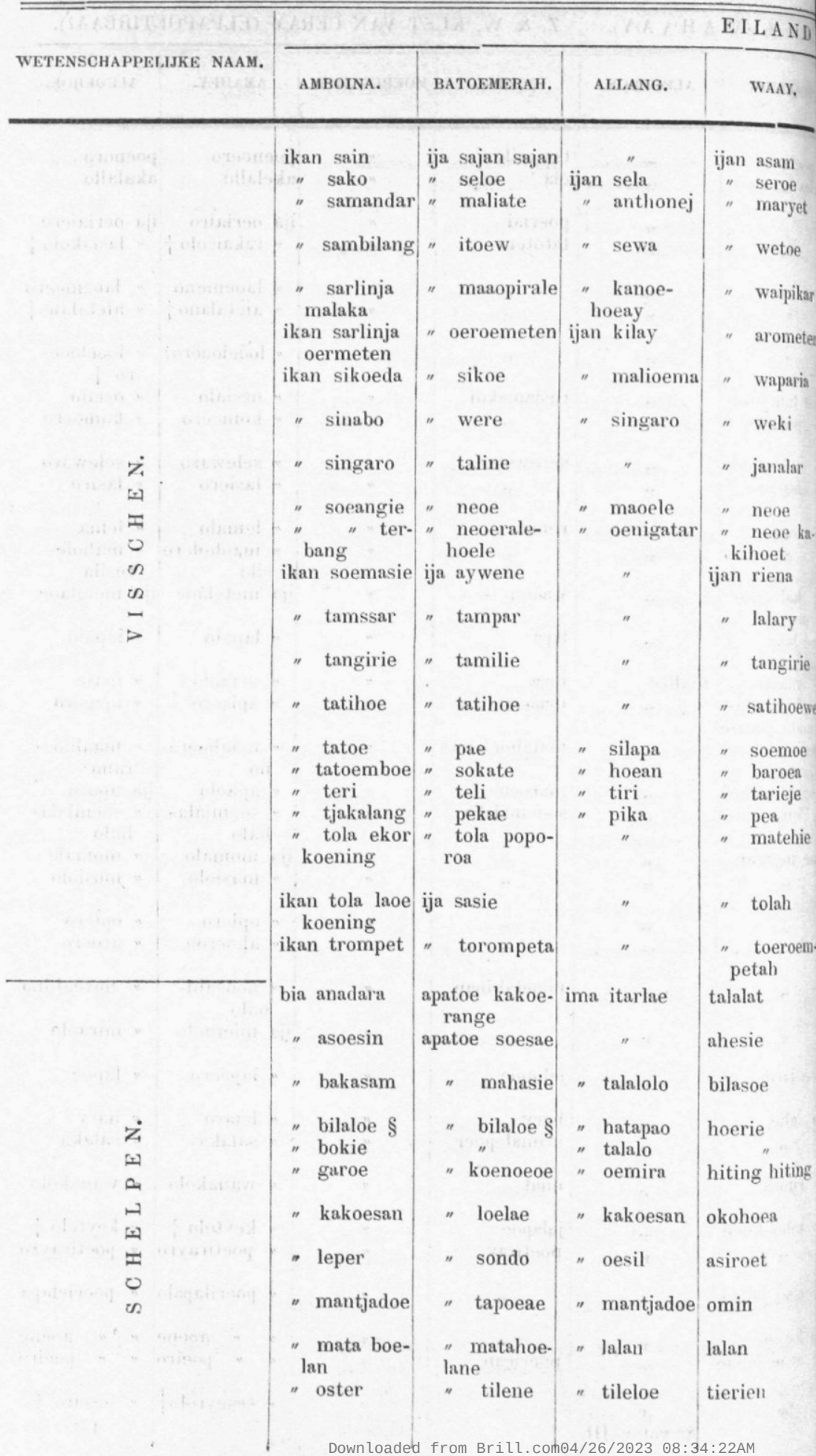




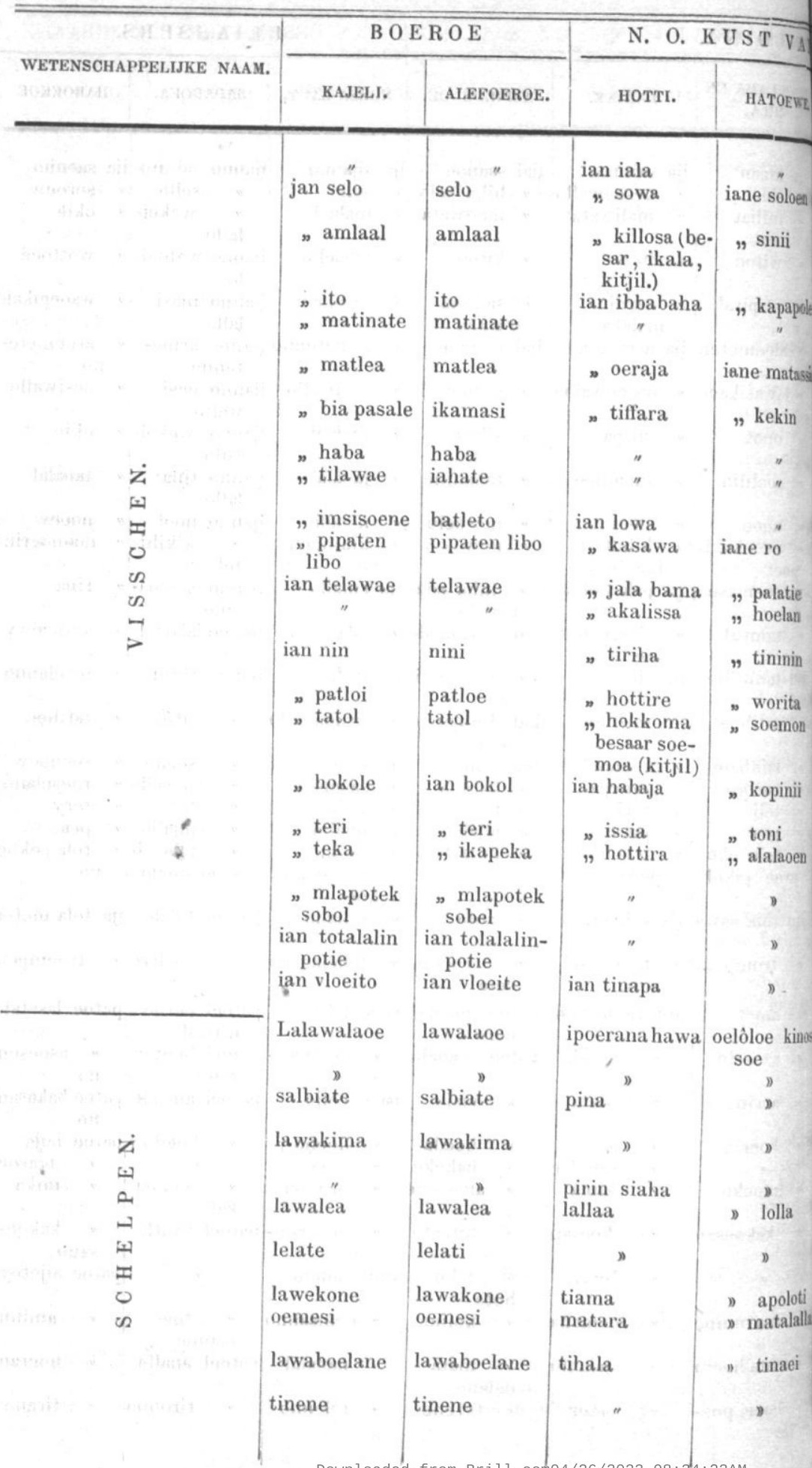




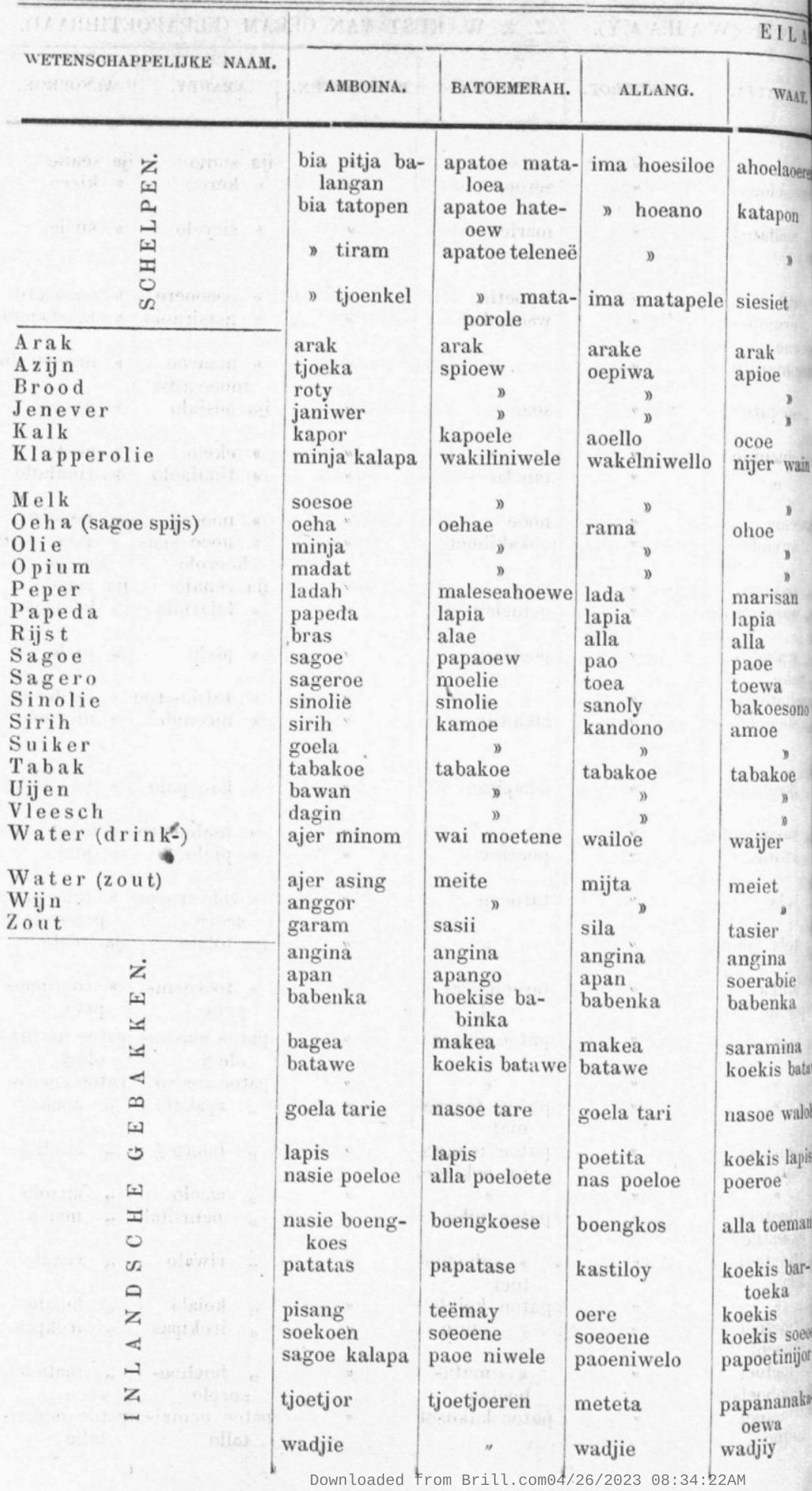




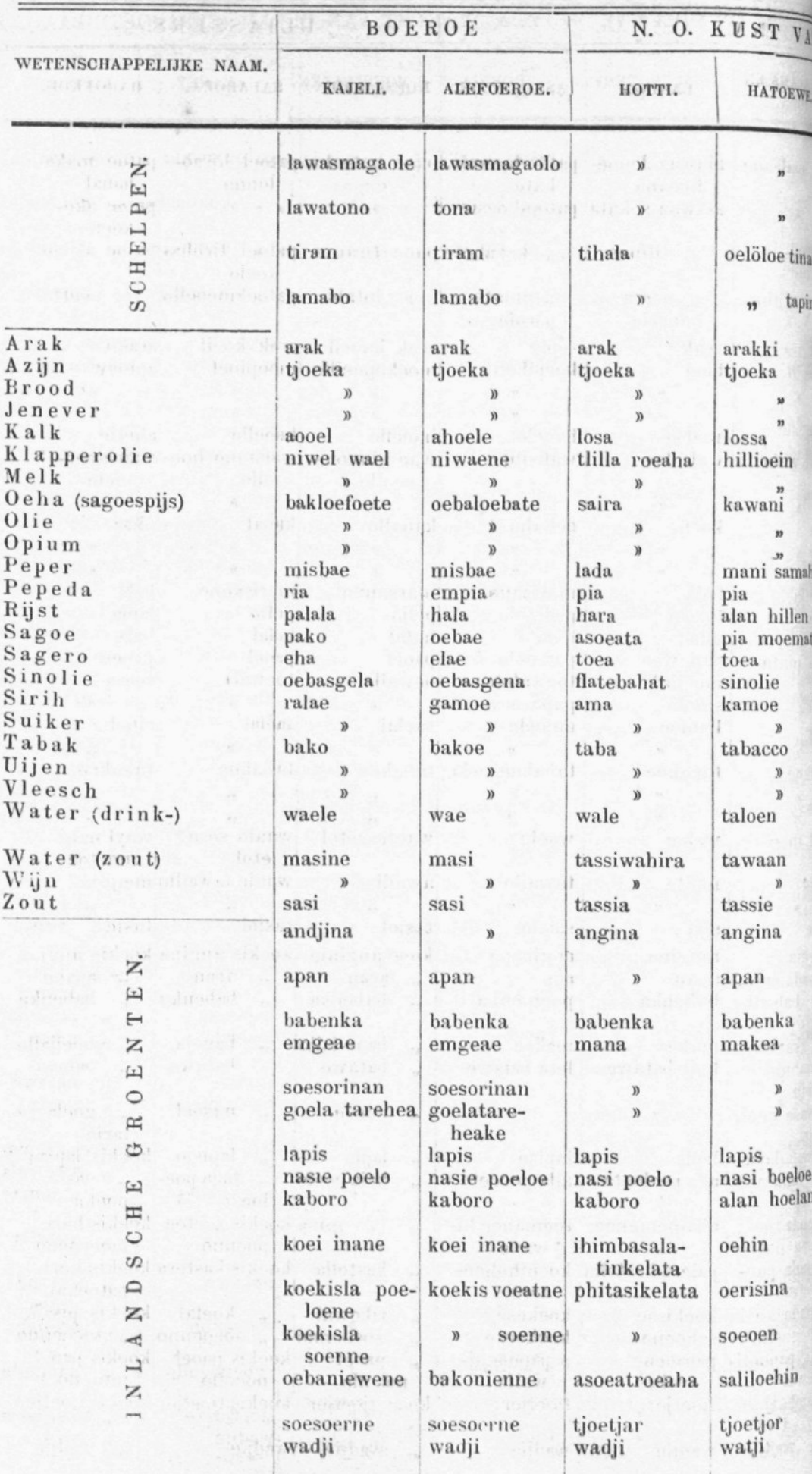


SCHETS VAN DE RESIDENTIE AMBOINA.

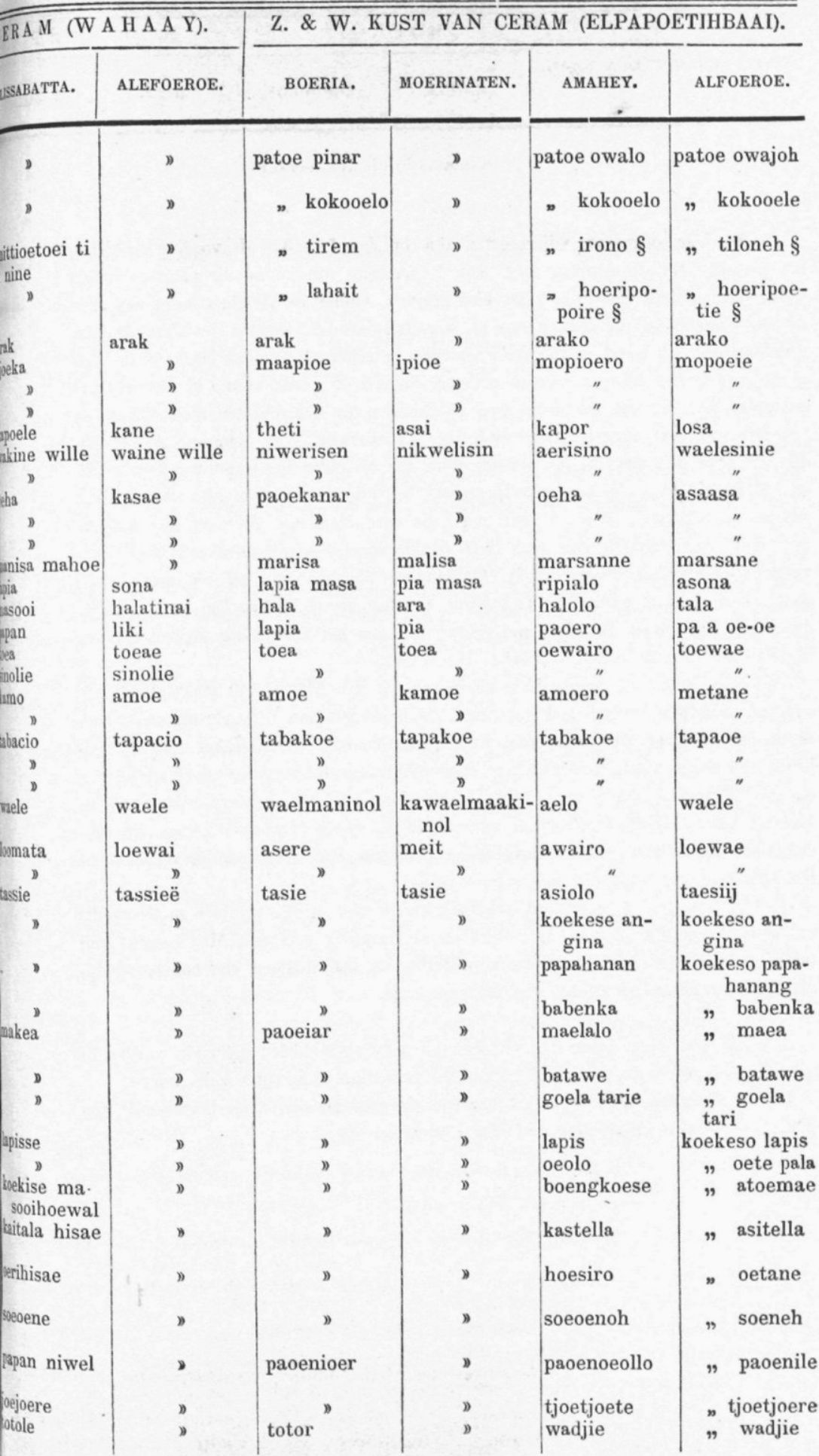




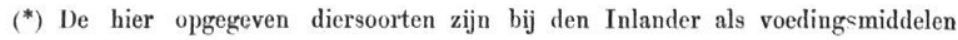
in gebruik. De diersoorten met een ${ }^{*}$ gemerkt worden op die plaatsen onder welke die aanteekening voorkomt niet gegeten. Onder de visschen loopt dat zeer uiteen. Visschen op de eene plats als vergiftig bekend, worden op andere plaatsen gegeten. Men beweert dat enkele visschen in zekere tijden des jaars, of in den paartijd vergiftig zijn, overigens eetbaar. Soms ook wordt hunne al of niet vergiftigheid afgeleid van de plaats van voorkomen. Er heerscht ten dien opzigte in het algemeen eene zeer betreurenswaardige onzekerheid. Vrij algemeen zoekt men de bron van het vergiftigend vermogen in een of ander ingewand van den visch.

$(\dagger)$ Ikan biba, algemeen als blaasvisch bekend, en waarvan hier verscheidene soorten voorkomen, wordt in den regel als voedingsmiddel gevreesd. Op Larieke zijn door het nuttigen van deze visch (welke species is mij oubekend) doodelijke vergiftigingsgevallen voorgekomen. Op andere plaatsen worden zij gegeten na vooraf hunne oerat verwijderd te hebben. Of deze oerat, gelijk Dr. Bleeker zegt, de ovaria zijn weet ik niet; wel echter dat men het zorgvuldig uituemen dezer ingewanden als eene eerste vereischte beschouwt.

De ikan komoe of komo die soms in scholen van 500 à 600 stuks wordt gevangen, wordt algemeen veel genuttigd. Men schrijft aan het gebruik van dezen visch het ontstaan der lepra toe. Van algemeene bekendheid is het, dat het gebruik van dezen visch, onder zekere omstandigheden, tot vergiftigingsverschijnselen aanleiding geeft, gelijk een geval in het hospitaal alhier werd waargenomen. Hoogst waarschijulijk ligt dan de oorzaak in het meer of min bedorven zijn vau den visch die in zoo groote hoeveelheden gevangen, dan niet dadelijk verkocht en ligt bewaard wordt tot den volgenden dag.

(\$) De met een $\S$ gemerkte schelpdieren worden op die platsen onder welke zulks is aangeteekend niet genuttigd en als vergiftig gevreesd. Men spreekt van meerdere gevallen van doodelijke vergiftiging na bet nuttigen van enkele schelpdieren, wier namen ik echter niet kon opsporen.

Alle in deze lijst genoemde visschen als voedingsmiddelen dienende worden òf gekookt, òf gedroogd, of veelal gcroosterd genuttigd. Somwijlen zelfs raauw.

In het algemeen heeft deze lijst, zoowel planten- als diereurijk betreffende, ten doel: kennis van de voedingsmiddelen des volks. 